\title{
Iterative Methods for Finding Common Solution of Generalized Equilibrium Problems and Variational Inequality Problems and Fixed Point Problems of a Finite Family of Nonexpansive Mappings
}

\begin{abstract}
Atid Kangtunyakarn
Department of Mathematics, Faculty of Science, King Mongkut's Institute of Technology Ladkrabang, Bangkok 10520, Thailand

Correspondence should be addressed to Atid Kangtunyakarn, beawrock@hotmail.com

Received 7 October 2010; Accepted 2 November 2010

Academic Editor: T. D. Benavides

Copyright (C) 2010 Atid Kangtunyakarn. This is an open access article distributed under the Creative Commons Attribution License, which permits unrestricted use, distribution, and reproduction in any medium, provided the original work is properly cited.

We introduce a new method for a system of generalized equilibrium problems, system of variational inequality problems, and fixed point problems by using $S$-mapping generated by a finite family of nonexpansive mappings and real numbers. Then, we prove a strong convergence theorem of the proposed iteration under some control condition. By using our main result, we obtain strong convergence theorem for finding a common element of the set of solution of a system of generalized equilibrium problems, system of variational inequality problems, and the set of common fixed points of a finite family of strictly pseudocontractive mappings.
\end{abstract}

\section{Introduction}

Let $H$ be a real Hilbert space, and let $C$ be a nonempty closed convex subset of $H$. Let $A$ : $C \rightarrow H$ be a nonlinear mapping, and let $F: C \times C \rightarrow \mathbb{R}$ be a bifunction. A mapping $T$ of $H$ into itself is called nonexpansive if $\|T x-T y\| \leq\|x-y\|$ for all $x, y \in H$. We denote by $F(T)$ the set of fixed points of $T$ (i.e., $F(T)=\{x \in H: T x=x\}$ ). Goebel and Kirk [1] showed that $F(T)$ is always closed convex, and also nonempty provided $T$ has a bounded trajectory.

A bounded linear operator $A$ on $H$ is called strongly positive with coefficient $\bar{\gamma}$ if there is a constant $\bar{\gamma}>0$ with the property

$$
\langle A x, x\rangle \geq \bar{\gamma}\|x\|^{2} .
$$


The equilibrium problem for $F$ is to find $x \in C$ such that

$$
F(x, y) \geq 0, \quad \forall y \in C
$$

The set of solutions of (1.2) is denoted by $\mathrm{EP}(F)$. Many problems in physics, optimization, and economics are seeking some elements of $\operatorname{EP}(F)$, see [2,3]. Several iterative methods have been proposed to solve the equilibrium problem, see, for instance, [2-4]. In 2005, Combettes and Hirstoaga [3] introduced an iterative scheme of finding the best approximation to the initial data when $\mathrm{EP}(F)$ is nonempty and proved a strong convergence theorem.

The variational inequality problem is to find a point $u \in C$ such that

$$
\langle v-u, \mathrm{~A} u\rangle \geq 0 \quad \forall v \in C .
$$

The set of solutions of the variational inequality is denoted by $\operatorname{VI}(C, A)$, and we consider the following generalized equilibrium problem.

Find $z \in C$ such that $F(z, y)+\langle A z, y-z\rangle \geq 0, \quad \forall y \in C$.

The set of such $z \in C$ is denoted by $\operatorname{EP}(F, A)$, that is,

$$
\mathrm{EP}(F, A)=\{z \in C: F(z, y)+\langle A z, y-z\rangle \geq 0, \forall y \in C\}
$$

In the case of $A \equiv 0, \mathrm{EP}(F, A)=\mathrm{EP}(F)$. Numerous problems in physics, optimization, variational inequalities, minimax problems, the Nash equilibrium problem in noncooperative games reduce to find element of (1.5)

A mapping $A$ of $C$ into $H$ is called inverse-strongly monotone, see [5], if there exists a positive real number $\alpha$ such that

$$
\langle x-y, A x-A y\rangle \geq \alpha\|A x-A y\|^{2}
$$

for all $x, y \in C$.

The problem of finding a common fixed point of a family of nonexpansive mappings has been studied by many authors. The well-known convex feasibility problem reduces to finding a point in the intersection of the fixed point sets of a family of nonexpansive mapping (see $[6,7])$.

The ploblem of finding a common element of $\operatorname{EP}(F, A)$ and the set of all common fixed points of a family of nonexpansive mappings is of wide interdisciplinary interest and importance. Many iterative methods are purposed for finding a common element of the solutions of the equilibrium problem and fixed point problem of nonexpansive mappings, see [8-10]. 
In 2008, S.Takahashi and W.Takahashi [11] introduced a general iterative method for finding a common element of $\operatorname{EP}(F, A)$ and $F(T)$. They defined $\left\{x_{n}\right\}$ in the following way:

$$
\begin{gathered}
u, x_{1} \in C, \quad \text { arbitrarily; } \\
F\left(z_{n}, y\right)+\left\langle A x_{n}, y-z_{n}\right\rangle+\frac{1}{\lambda_{n}}\left\langle y-z_{n}, z_{n}-x_{n}\right\rangle \geq 0, \quad \forall y \in C, \\
x_{n+1}=\beta_{n} x_{n}+\left(1-\beta_{n}\right) T\left(a_{n} u+\left(1-a_{n}\right) z_{n}\right), \quad \forall n \in \mathbb{N},
\end{gathered}
$$

where $A$ is an $\alpha$-inverse strongly monotone mapping of $C$ into $H$ with positive real number $\alpha$, and $\left\{a_{n}\right\} \in[0,1],\left\{\beta_{n}\right\} \subset[0,1],\left\{\lambda_{n}\right\} \subset[0,2 \alpha]$, and proved strong convergence of the scheme (1.7) to $z \in \bigcap_{i=1}^{N} F\left(T_{i}\right) \cap \operatorname{EP}(F, A)$, where $z=P_{\bigcap_{i=1}^{N} F\left(T_{i}\right) \cap \operatorname{EP}(F, A)} u$ in the framework of a Hilbert space, under some suitable conditions on $\left\{a_{n}\right\},\left\{\beta_{n}\right\},\left\{\lambda_{n}\right\}$ and bifunction $F$.

Very recently, in 2010, Qin, et al. [12] introduced a iterative scheme method for finding a common element of $\operatorname{EP}\left(F_{1}, A\right), \operatorname{EP}\left(F_{2}, B\right)$ and common fixed point of infinite family of nonexpansive mappings. They defined $\left\{x_{n}\right\}$ in the following way:

$$
\begin{gathered}
x_{1} \in C, \quad \text { arbitrarily; } \\
F_{1}\left(u_{n}, u\right)+\left\langle A x_{n}, u-u_{n}\right\rangle+\frac{1}{r}\left\langle u-u_{n}, u_{n}-x_{n}\right\rangle \geq 0, \quad \forall u \in C, \\
F_{2}\left(v_{n}, v\right)+\left\langle B x_{n}, v-v_{n}\right\rangle+\frac{1}{S}\left\langle v-v_{n}, v_{n}-x_{n}\right\rangle \geq 0, \quad \forall v \in C, \\
y_{n}=\delta_{n} u_{n}+\left(1-\delta_{n}\right) v_{n}, \\
x_{n+1}=\alpha_{n} f\left(x_{n}\right)+\beta_{n} x_{n}+\gamma_{n} W_{n} x_{n}, \quad \forall n \in \mathbb{N},
\end{gathered}
$$

where $f: C \rightarrow C$ is a contraction mapping and $W_{n}$ is $W$-mapping generated by infinite family of nonexpansive mappings and infinite real number. Under suitable conditions of these parameters they proved strong convergence of the scheme (1.8) to $z=P_{\mathfrak{F}} f(z)$, where $\mathfrak{F}=\bigcap_{i=1}^{\infty} F\left(T_{i}\right) \cap \operatorname{EP}\left(F_{1}, A\right) \cap \operatorname{EP}\left(F_{2}, B\right)$. defined by

In this paper, motivated by $[11,12]$, we introduced a general iterative scheme $\left\{x_{n}\right\}$

$$
\begin{gathered}
F\left(u_{n}, u\right)+\left\langle A x_{n}, u-u_{n}\right\rangle+\frac{1}{r_{n}}\left\langle u-u_{n}, u_{n}-x_{n}\right\rangle \geq 0, \\
G\left(v_{n}, v\right)+\left\langle B x_{n}, v-v_{n}\right\rangle+\frac{1}{S_{n}}\left\langle v-v_{n}, v_{n}-x_{n}\right\rangle \geq 0, \\
y_{n}=\delta_{n} P_{C}\left(u_{n}-\lambda_{n} A u_{n}\right)+\left(1-\delta_{n}\right) P_{C}\left(v_{n}-\eta_{n} B v_{n}\right), \\
x_{n+1}=\alpha_{n} f\left(x_{n}\right)+\beta_{n} x_{n}+\gamma_{n} S_{n} y_{n}, \quad \forall n \geq 0,
\end{gathered}
$$


where $f: C \rightarrow C$ and $S_{n}$ is $S$-mapping generated by $T_{0}, \ldots, T_{n}$ and $\alpha_{n}, \alpha_{n-1}, \ldots, \alpha_{0}$. Under suitable conditions, we proved strong convergence of $\left\{x_{n}\right\}$ to $z=P_{\mathfrak{F}} f(z)$, and $z$ is solution of

$$
\begin{aligned}
& \left\langle A x^{*}, x-x^{*}\right\rangle \geq 0, \\
& \left\langle B x^{*}, x-x^{*}\right\rangle \geq 0 .
\end{aligned}
$$

\section{Preliminaries}

In this section, we collect and give some useful lemmas that will be used for our main result in the next section.

Let $C$ be closed convex subset of a real Hilbert space $H$, and let $P_{C}$ be the metric projection of $H$ onto $C$, that is, for $x \in H, P_{C} x$ satisfies the property

$$
\left\|x-P_{C} x\right\|=\min _{y \in C}\|x-y\|
$$

The following characterizes the projection $P_{C}$.

Lemma 2.1 (see [13]). Given $x \in H$ and $y \in C$. Then $P_{C} x=y$ if and only if there holds the inequality

$$
\langle x-y, y-z\rangle \geq 0 \quad \forall z \in C
$$

Lemma 2.2 (see [14]). Let $\left\{s_{n}\right\}$ be a sequence of nonnegative real numbers satisfying

$$
s_{n+1}=\left(1-\alpha_{n}\right) s_{n}+\beta_{n}, \quad \forall n \geq 0
$$

where $\left\{\alpha_{n}\right\},\left\{\beta_{n}\right\}$ satisfy the conditions

(1) $\left\{\alpha_{n}\right\} \subset(0,1), \sum_{n=1}^{\infty} \sum \alpha_{n}=\infty$,

(2) $\lim \sup _{n \rightarrow \infty} \beta_{n} / \alpha_{n} \leq 0$.

Then $\lim _{n \rightarrow \infty} s_{n}=0$.

Lemma 2.3 (see [15]). Let $C$ be a closed convex subset of a strictly convex Banach space E. Let $\left\{T_{n}: n \in \mathbb{N}\right\}$ be a sequence of nonexpansive mappings on $C$. Suppose that $\bigcap_{n=1}^{\infty} F\left(T_{n}\right)$ is nonempty. Let $\left\{\lambda_{n}\right\}$ be a sequence of positive numbers with $\sum_{n=1}^{\infty} \lambda_{n}=1$. Then a mapping $S$ on $C$ defined by

$$
S(x)=\sum_{n=1}^{\infty} \lambda_{n} T_{n} x
$$

for $x \in C$ is well defined, nonexpansive, and $F(S)=\bigcap_{n=1}^{\infty} F\left(T_{n}\right)$ hold.

Lemma 2.4 (see [16]). Let E be a uniformly convex Banach space, $C$ a nonempty closed convex subset of $E$, and $S: C \rightarrow C$ a nonexpansive mapping. Then $I-S$ is demiclosed at zero. 
Lemma 2.5 (see [17]). Let $\left\{x_{n}\right\}$ and $\left\{z_{n}\right\}$ be bounded sequences in a Banach space $X$, and let $\left\{\beta_{n}\right\}$ be a sequence in $[0,1]$ with $0<\liminf _{n \rightarrow \infty} \beta_{n} \leq \lim \sup _{n \rightarrow \infty} \beta_{n}<1$. Suppose that

$$
x_{n+1}=\beta_{n} x_{n}+\left(1-\beta_{n}\right) z_{n}
$$

for all integer $n \geq 0$ and

$$
\lim \sup _{n \rightarrow \infty}\left(\left\|z_{n+1}-z_{n}\right\|-\left\|x_{n+1}-x_{n}\right\|\right) \leq 0
$$

Then $\lim _{n \rightarrow \infty}\left\|x_{n}-z_{n}\right\|=0$.

For solving the equilibrium problem for a bifunction $F: C \times C \rightarrow \mathbb{R}$, let us assume that $F$ satisfies the following conditions:

(A1) $F(x, x)=0$ for all $x \in C$;

(A2) $F$ is monotone, that is, $F(x, y)+F(y, x) \leq 0, \quad \forall x, y \in C$,

(A3) for all $x, y, z \in C$,

$$
\lim _{t \rightarrow 0^{+}} F(t z+(1-t) x, y) \leq F(x, y)
$$

(A4) for all $x \in C, y \mapsto F(x, y)$ is convex and lower semicontinuous.

The following lemma appears implicitly in [2].

Lemma 2.6 (see [2]). Let $C$ be a nonempty closed convex subset of $H$, and let $F$ be a bifunction of $C \times C$ into $\mathbb{R}$ satisfying (A1)-(A4). Let $r>0$ and $x \in H$. Then, there exists $z \in C$ such that

$$
F(z, y)+\frac{1}{r}\langle y-z, z-x\rangle
$$

for all $x \in C$.

Lemma 2.7 (see [3]). Assume that $F: C \times C \rightarrow \mathbb{R}$ satisfies (A1)-(A4). For $r>0$ and $x \in H$, define a mapping $T_{r}: H \rightarrow C$ as follows:

$$
T_{r}(x)=\left\{z \in C: F(z, y)+\frac{1}{r}\langle y-z, z-x\rangle \geq 0, \forall y \in C\right\} .
$$

for all $z \in H$. Then, the following hold:

(1) $T_{r}$ is single-valued;

(2) $T_{r}$ is firmly nonexpansive, that is,

$$
\left\|T_{r}(x)-T_{r}(y)\right\|^{2} \leq\left\langle T_{r}(x)-T_{r}(y), x-y\right\rangle \quad \forall x, y \in H ;
$$

(3) $F\left(T_{r}\right)=\mathrm{EP}(F)$;

(4) $\mathrm{EP}(F)$ is closed and convex. 
In 2009, Kangtunyakarn and Suantai [18] defined a new mapping and proved their lemma as follows.

Definition 2.8. Let $C$ be a nonempty convex subset of real Banach space. Let $\left\{T_{i}\right\}_{i=1}^{N}$ be a finite family of nonexpansive mappings of $C$ into itself. For each $j=1,2, \ldots, N$, let $\alpha_{j}=\left(\alpha_{1}^{j}, \alpha_{2}^{j}, \alpha_{3}^{j}\right) \in$ $I \times I \times I$, where $I \in[0,1]$ and $\alpha_{1}^{j}+\alpha_{2}^{j}+\alpha_{3}^{j}=1$. We define the mapping $S: C \rightarrow C$ as follows:

$$
\begin{aligned}
U_{0} & =I \\
U_{1} & =\alpha_{1}^{1} T_{1} U_{0}+\alpha_{2}^{1} U_{0}+\alpha_{3}^{1} I, \\
U_{2} & =\alpha_{1}^{2} T_{2} U_{1}+\alpha_{2}^{2} U_{1}+\alpha_{3}^{2} I, \\
U_{3} & =\alpha_{1}^{3} T_{3} U_{2}+\alpha_{2}^{3} U_{2}+\alpha_{3}^{3} I, \\
& \vdots \\
U_{N-1} & =\alpha_{1}^{N-1} T_{N-1} U_{N-2}+\alpha_{2}^{N-1} U_{N-2}+\alpha_{3}^{N-1} I, \\
S & =U_{N}=\alpha_{1}^{N} T_{N} U_{N-1}+\alpha_{2}^{N} U_{N-1}+\alpha_{3}^{N} I .
\end{aligned}
$$

This mapping is called $S$-mapping generated by $T_{1}, \ldots, T_{N}$ and $\alpha_{1}, \alpha_{2}, \ldots, \alpha_{N}$.

Lemma 2.9. Let $C$ be a nonempty closed convex subset of strictly convex. Let $\left\{T_{i}\right\}_{i=1}^{N}$ be a finite family of nonexpanxive mappings of $C$ into itself with $\bigcap_{i=1}^{N} F\left(T_{i}\right) \neq \emptyset$, and let $\alpha_{j}=\left(\alpha_{1}^{j}, \alpha_{2}^{j}, \alpha_{3}^{j}\right) \in I \times I \times I$, $j=1,2,3, \ldots, N$, where $I=[0,1], \alpha_{1}^{j}+\alpha_{2}^{j}+\alpha_{3}^{j}=1, \alpha_{1}^{j} \in(0,1)$ for all $j=1,2, \ldots, N-1, \alpha_{1}^{N} \in$ $(0,1] \alpha_{2}^{j}, \alpha_{3}^{j} \in[0,1)$ for all $j=1,2, \ldots, N$. Let $S$ be the mapping generated by $T_{1}, \ldots, T_{N}$ and $\alpha_{1}, \alpha_{2}, \ldots, \alpha_{N}$. Then $F(S)=\bigcap_{i=1}^{N} F\left(T_{i}\right)$.

Lemma 2.10. Let $C$ be a nonempty closed convex subset of Banach space. Let $\left\{T_{i}\right\}_{i=1}^{N}$ be a finite family of nonexpansive mappings of $C$ into itself and $\alpha_{j}^{(n)}=\left(\alpha_{1}^{n, j}, \alpha_{2}^{n, j}, \alpha_{3}^{n, j}\right), \alpha_{j}=\left(\alpha_{1}^{j}, \alpha_{2}^{j}, \alpha_{3}^{j}\right) \in I \times I \times I$, where $I=[0,1], \alpha_{1}^{n, j}+\alpha_{2}^{n, j}+\alpha_{3}^{n, j}=1$ and $\alpha_{1}^{j}+\alpha_{2}^{j}+\alpha_{3}^{j}=1$ such that $\alpha_{i}^{n, j} \rightarrow \alpha_{i}^{j} \in[0,1]$ as $n \rightarrow$ $\infty$ for $i=1,3$ and $j=1,2,3, \ldots, N$. Moreover, for every $n \in \mathbb{N}$, let $S$ and $S_{n}$ be the $S$-mappings generated by $T_{1}, T_{2}, \ldots, T_{N}$ and $\alpha_{1}, \alpha_{2}, \ldots, \alpha_{N}$ and $T_{1}, T_{2}, \ldots, T_{N}$ and $\alpha_{1}^{(n)}, \alpha_{2}^{(n)}, \ldots, \alpha_{N}^{(n)}$, respectively. Then $\lim _{n \rightarrow \infty}\left\|S_{n} x-S x\right\|=0$ for every $x \in C$.

Lemma 2.11 (see [19]). Let $C$ be a nonempty closed convex subset of a Hilbert space $H$, and let $G: C \rightarrow C$ be defined by

$$
G(x)=P_{C}(x-\lambda A x), \quad \forall x \in C,
$$

with $\forall \lambda>0$. Then $x^{*} \in V I(C, A)$ if and only if $x^{*} \in F(G)$.

\section{Main Result}

Theorem 3.1. Let $C$ be a nonempty closed convex subset of a Hilbert space $H$. Let $F$ and $G$ be two bifunctions from $C \times C$ into $\mathbb{R}$ satisfying (A1)-(A4), respectively. Let $A: C \rightarrow H$ a $\alpha$-inverse strongly monotone mapping and $B: C \rightarrow H$ be a $\beta$-inverse strongly monotone mapping. Let $\left\{T_{i}\right\}_{i=1}^{N}$ be finite 
family of nonexpansive mappings with $\mathfrak{F}=\bigcap_{i=1}^{N} F\left(T_{i}\right) \cap \operatorname{EP}(F, A) \cap \operatorname{EP}(G, B) \cap F\left(G_{1}\right) \cap F\left(G_{2}\right) \neq \emptyset$, where $G_{1}, G_{2}: C \rightarrow C$ are defined by $G_{1}(x)=P_{C}\left(x-\lambda_{n} A x\right), G_{2}(x)=P_{C}\left(x-\eta_{n} B x\right), \forall x \in C$. Let $f: C \rightarrow C$ be a contraction with the coefficient $\theta \in(0,1)$. Let $S_{n}$ be the S-mappings generated by $T_{1}, T_{2}, \ldots, T_{N}$ and $\alpha_{1}^{(n)}, \alpha_{2}^{(n)}, \ldots, \alpha_{N}^{(n)}$, where $\alpha_{j}^{(n)}=\left(\alpha_{1}^{n, j}, \alpha_{2}^{n, j}, \alpha_{3}^{n, j}\right) \in I \times I \times I, I=[0,1], \alpha_{1}^{n, j}+$ $\alpha_{2}^{n, j}+\alpha_{3}^{n, j}=1$ and $0<\eta_{1} \leq \alpha_{1}^{n, j} \leq \theta_{1}<1 \forall n \in \mathbb{N}, \forall j=1,2, \ldots, N-1,0<\eta_{N} \leq \alpha_{1}^{n, N} \leq 1$ and $0 \leq \alpha_{2}^{n, j}, \alpha_{3}^{n, j} \leq \theta_{3}<1 \forall n \in \mathbb{N}, \forall j=1,2, \ldots, N$. Let $\left\{x_{n}\right\},\left\{u_{n}\right\},\left\{v_{n}\right\},\left\{y_{n}\right\}$ be sequences generated by $x_{1}, u, v \in C$

$$
\begin{gathered}
F\left(u_{n}, u\right)+\left\langle A x_{n}, u-u_{n}\right\rangle+\frac{1}{r_{n}}\left\langle u-u_{n}, u_{n}-x_{n}\right\rangle \geq 0, \\
G\left(v_{n}, v\right)+\left\langle B x_{n}, v-v_{n}\right\rangle+\frac{1}{S_{n}}\left\langle v-v_{n}, v_{n}-x_{n}\right\rangle \geq 0, \\
y_{n}=\delta_{n} P_{C}\left(u_{n}-\lambda_{n} A u_{n}\right)+\left(1-\delta_{n}\right) P_{C}\left(v_{n}-\eta_{n} B v_{n}\right), \\
x_{n+1}=\alpha_{n} f\left(x_{n}\right)+\beta_{n} x_{n}+\gamma_{n} S_{n} y_{n}, \quad \forall n \geq 1,
\end{gathered}
$$

where $\left\{\alpha_{n}\right\},\left\{\beta_{n}\right\},\left\{\gamma_{n}\right\} \in(0,1)$ such that $\alpha_{n}+\beta_{n}+\gamma_{n}=1, r_{n} \in[a, b] \subset(0,2 \alpha), s_{n} \in[c, d] \subset$ $(0,2 \beta), \lambda_{n} \in[e, f] \subset(0,2 \alpha), \eta_{n} \in[g, h] \subset(0,2 \beta)$. Assume that

(i) $\lim _{n \rightarrow \infty} n=0$ and $\sum_{n=0}^{\infty} \alpha_{n}=\infty$,

(ii) $0<\liminf _{n \rightarrow \infty} \beta_{n} \leq \lim \sup _{n \rightarrow \infty} \beta_{n}<1$,

(iii) $\lim _{n \rightarrow \infty} \delta_{n}=\delta \in(0,1)$,

(iv) $\sum_{n=0}^{\infty}\left|s_{n+1}-s_{n}\right|, \sum_{n=0}^{\infty}\left|r_{n+1}-r_{n}\right|, \sum_{n=0}^{\infty}\left|\lambda_{n+1}-\lambda_{n}\right|, \sum_{n=0}^{\infty}\left|\eta_{n+1}-\eta_{n}\right|, \sum_{n=0}^{\infty}\left|\alpha_{n+1}-\alpha_{n}\right|, \sum_{n=0}^{\infty} \mid \beta_{n+1}-$ $\beta_{n} \mid<\infty$,

(v) $\left|\alpha_{1}^{n+1, j}-\alpha_{1}^{n, j}\right| \rightarrow 0$, and $\left|\alpha_{3}^{n+1, j}-\alpha_{3}^{n, j}\right| \rightarrow 0$ as $n \rightarrow \infty$, for all $j \in\{1,2,3, \ldots, N\}$.

Then the sequence $\left\{x_{n}\right\},\left\{y_{n}\right\},\left\{u_{n}\right\},\left\{v_{n}\right\}$ converge strongly to $z=P_{\mathfrak{F}} f(z)$, and $z$ is solution of

$$
\begin{aligned}
& \left\langle A x^{*}, x-x^{*}\right\rangle \geq 0, \\
& \left\langle B x^{*}, x-x^{*}\right\rangle \geq 0 .
\end{aligned}
$$

Proof. First, we show that $\left(I-\lambda_{n} A\right),\left(I-\eta_{n} B\right)\left(I-r_{n} A\right)$ and $\left(I-s_{n} B\right)$ are nonexpansive. Let $x, y \in C$. Since $A$ is $\alpha$-strongly monotone and $\lambda_{n}<2 \alpha$ for all $n \in \mathbb{N}$, we have

$$
\begin{aligned}
\left\|\left(I-\lambda_{n} A\right) x-\left(I-\lambda_{n} A\right) y\right\|^{2} & =\left\|x-y-\lambda_{n}(A x-A y)\right\|^{2} \\
& =\|x-y\|^{2}-2 \lambda_{n}\langle x-y, A x-A y\rangle+\lambda_{n}^{2}\|A x-A y\|^{2} \\
& \leq\|x-y\|^{2}-2 \alpha \lambda_{n}\|A x-A y\|^{2}+\lambda_{n}^{2}\|A x-A y\|^{2} \\
& =\|x-y\|^{2}+\lambda_{n}\left(\lambda_{n}-2 \alpha\right)\|A x-A y\|^{2} \\
& \leq\|x-y\|^{2} .
\end{aligned}
$$


Thus $\left(I-\lambda_{n} A\right)$ is nonexpansive. By using the same proof, we obtain that $\left(I-\eta_{n} B\right)\left(I-r_{n} A\right)$ and $\left(I-s_{n} B\right)$ are nonexpansive.

We will divide our proof into 6 steps.

Step 1 . We will show that the sequence $\left\{x_{n}\right\}$ is bounded. Since

$$
F\left(u_{n}, u\right)+\left\langle A x_{n}, u-u_{n}\right\rangle+\frac{1}{r_{n}}\left\langle u-u_{n}, u_{n}-x_{n}\right\rangle \geq 0, \quad \forall u \in C,
$$

then we have

$$
F\left(u_{n}, u\right)+\frac{1}{r_{n}}\left\langle u-u_{n}, u_{n}-\left(I-r_{n} A\right) x_{n}\right\rangle \geq 0
$$

By Lemma 2.7, we have $u_{n}=T_{r_{n}}\left(I-r_{n} A\right) x_{n}$. By the same argument as above, we obtaine that $v_{n}=T_{s_{n}}\left(I-s_{n} B\right) x_{n}$

Let $z \in \mathfrak{F}$. Then $F(z, y)+\langle y-z, A z\rangle \geq 0$ and $G(z, y)+\langle y-z, B z\rangle \geq 0$. Hence

$$
\begin{aligned}
& F(z, y)+\frac{1}{r_{n}}\left\langle y-z, z-z+r_{n} A z\right\rangle \geq 0, \\
& G(z, y)+\frac{1}{s_{n}}\left\langle y-z, z-z+s_{n} B z\right\rangle \geq 0 .
\end{aligned}
$$

Again by Lemma 2.7, we have $z=T_{r_{n}}\left(z-r_{n} A z\right)=T_{S_{n}}\left(z-s_{n} B z\right)$. Since $z \in \mathfrak{F}$, we have $z=P_{C}\left(I-\lambda_{n} A\right) z=P_{C}\left(I-\eta_{n} B\right) z$. By nonexpansiveness of $T_{r_{n}}, T_{s_{n}}, I-r_{n} A, I-s_{n} B$, we have

$$
\begin{aligned}
\left\|x_{n+1}-z\right\| \leq & \alpha_{n}\left\|f\left(x_{n}\right)-z\right\|+\beta_{n}\left\|x_{n}-z\right\|+\gamma_{n}\left\|S_{n} y_{n}-z\right\| \\
\leq & \alpha_{n}\left\|f\left(x_{n}\right)-f(z)\right\|+\alpha_{n}\|f(z)-z\|+\beta_{n}\left\|x_{n}-z\right\|+\gamma_{n}\left\|y_{n}-z\right\| \\
\leq & \alpha_{n} \theta\left\|x_{n}-z\right\|+\alpha_{n}\|f(z)-z\|+\beta_{n}\left\|x_{n}-z\right\| \\
& +\gamma_{n}\left\|\delta_{n}\left(P_{C}\left(u_{n}-\lambda_{n} A u_{n}\right)-z\right)+\left(1-\delta_{n}\right)\left(P_{C}\left(v_{n}-\eta_{n} B v_{n}\right)-z\right)\right\| \\
\leq & \alpha_{n} \theta\left\|x_{n}-z\right\|+\alpha_{n}\|f(z)-z\|+\beta_{n}\left\|x_{n}-z\right\|+\gamma_{n}\left(\delta_{n}\left\|u_{n}-z\right\|+\left(1-\delta_{n}\right)\left\|v_{n}-z\right\|\right) \\
= & \alpha_{n} \theta\left\|x_{n}-z\right\|+\alpha_{n}\|f(z)-z\|+\beta_{n}\left\|x_{n}-z\right\| \\
& +\gamma_{n}\left(\delta_{n}\left\|T_{r_{n}}\left(I-r_{n} A\right) x_{n}-T_{r_{n}}\left(I-r_{n} A\right) z\right\|\right. \\
& \left.+\left(1-\delta_{n}\right)\left\|T_{S_{n}}\left(I-s_{n} B\right) x_{n}-T_{S_{n}}\left(I-s_{n} B\right) z\right\|\right) \\
\leq & \alpha_{n} \theta\left\|x_{n}-z\right\|+\alpha_{n}\|f(z)-z\|+\beta_{n}\left\|x_{n}-z\right\|+\gamma_{n}\left\|x_{n}-z\right\| \\
= & \alpha_{n} \theta\left\|x_{n}-z\right\|+\alpha_{n}\|f(z)-z\|+\left(1-\alpha_{n}\right)\left\|x_{n}-z\right\| \\
= & \left(1-\alpha_{n}(1-\theta)\right)\left\|x_{n}-z\right\|+\alpha_{n}\|f(z)-z\| \\
\leq & \max \left\{\left\|x_{n}-z\right\|, \frac{\|f(z)-z\|}{1-\theta}\right\} .
\end{aligned}
$$


By induction we can prove that $\left\{x_{n}\right\}$ is bounded and so are $\left\{u_{n}\right\},\left\{v_{n}\right\},\left\{y_{n}\right\},\left\{S_{n} y_{n}\right\}$. Without of generality, assume that there exists a bounded set $K \subset C$ such that

$$
\left\{u_{n}\right\},\left\{v_{n}\right\},\left\{y_{n}\right\},\left\{S_{n} y_{n}\right\} \in K
$$

Step 2. We will show that $\lim _{n \rightarrow \infty}\left\|x_{n+1}-x_{n}\right\|=0$.

Putting $k_{n}=\left(x_{n+1}-\beta_{n} x_{n}\right) /\left(1-\beta_{n}\right)$, we have

$$
x_{n+1}=\left(1-\beta_{n}\right) k_{n}+\beta_{n} x_{n}, \quad \forall n \geq 0 .
$$

From definition of $k_{n}$, we have

$$
\begin{aligned}
\left\|k_{n+1}-k_{n}\right\|= & \left\|\frac{x_{n+2}-\beta_{n+1} x_{n+1}}{1-\beta_{n+1}}-\frac{x_{n+1}-\beta_{n} x_{n}}{1-\beta_{n}}\right\| \\
= & \left\|\frac{\alpha_{n+1} f\left(x_{n+1}\right)+\gamma_{n+1} S_{n+1} y_{n+1}}{1-\beta_{n+1}}-\frac{\alpha_{n} f\left(x_{n}\right)+\gamma_{n} S_{n} y_{n}}{1-\beta_{n}}\right\| \\
= & \left\|\frac{\alpha_{n+1} f\left(x_{n+1}\right)+\left(1-\beta_{n+1}-\alpha_{n+1}\right) S_{n+1} y_{n+1}}{1-\beta_{n+1}}-\frac{\alpha_{n} f\left(x_{n}\right)+\left(1-\beta_{n}-\alpha_{n}\right) S_{n} y_{n} \|}{1-\beta_{n}}\right\| \\
= & \left\|\frac{\alpha_{n+1}}{1-\beta_{n+1}}\left(f\left(x_{n+1}\right)-S_{n+1} y_{n+1}\right)-\frac{\alpha_{n}}{1-\beta_{n}}\left(f\left(x_{n}\right)-S_{n} y_{n}\right)+S_{n+1} y_{n+1}-S_{n} y_{n}\right\| \\
\leq & \frac{\alpha_{n+1}}{1-\beta_{n+1}}\left\|f\left(x_{n+1}\right)-S_{n+1} y_{n+1}\right\|+\frac{\alpha_{n}}{1-\beta_{n}}\left\|f\left(x_{n}\right)-S_{n} y_{n}\right\|+\left\|S_{n+1} y_{n+1}-S_{n} y_{n}\right\| \\
\leq & \frac{\alpha_{n+1}}{1-\beta_{n+1}}\left\|f\left(x_{n+1}\right)-S_{n+1} y_{n+1}\right\|+\frac{\alpha_{n}}{1-\beta_{n}}\left\|f\left(x_{n}\right)-S_{n} y_{n}\right\| \\
& +\left\|S_{n+1} y_{n+1}-S_{n} y_{n}\right\|+\left\|x_{n+1}-x_{n}\right\| .
\end{aligned}
$$


By definition of $S_{n}$, for $k \in\{2,3, \ldots, N\}$, we have

$$
\begin{aligned}
& \left\|U_{n+1, k} y_{n}-U_{n, k} y_{n}\right\|=\| \alpha_{1}^{n+1, k} T_{k} U_{n+1, k-1} y_{n}+\alpha_{2}^{n+1, k} U_{n+1, k-1} y_{n}+\alpha_{3}^{n+1, k} y_{n} \\
& -\alpha_{1}^{n, k} T_{k} U_{n, k-1} y_{n}-\alpha_{2}^{n, k} U_{n, k-1} y_{n}-\alpha_{3}^{n, k} y_{n} \| \\
& =\| \alpha_{1}^{n+1, k}\left(T_{k} U_{n+1, k-1} y_{n}-T_{k} U_{n, k-1} y_{n}\right)+\left(\alpha_{1}^{n+1, k}-\alpha_{1}^{n, k}\right) T_{k} U_{n, k-1} y_{n} \\
& +\left(\alpha_{3}^{n+1, k}-\alpha_{3}^{n, k}\right) y_{n}+\alpha_{2}^{n+1, k}\left(U_{n+1, k-1} y_{n}-U_{n, k-1} y_{n}\right) \\
& +\left(\alpha_{2}^{n+1, k}-\alpha_{2}^{n, k}\right) U_{n, k-1} y_{n} \| \\
& \leq \alpha_{1}^{n+1, k}\left\|U_{n+1, k-1} y_{n}-U_{n, k-1} y_{n}\right\|+\left|\alpha_{1}^{n+1, k}-\alpha_{1}^{n, k}\right|\left\|T_{k} U_{n, k-1} y_{n}\right\| \\
& +\left|\alpha_{3}^{n+1, k}-\alpha_{3}^{n, k}\right|\left\|y_{n}\right\|+\alpha_{2}^{n+1, k}\left\|U_{n+1, k-1} y_{n}-U_{n, k-1} y_{n}\right\| \\
& +\left|\alpha_{2}^{n+1, k}-\alpha_{2}^{n, k}\right|\left\|U_{n, k-1} y_{n}\right\| \\
& =\left(\alpha_{1}^{n+1, k}+\alpha_{2}^{n+1, k}\right)\left\|U_{n+1, k-1} y_{n}-U_{n, k-1} y_{n}\right\| \\
& +\left|\alpha_{1}^{n+1, k}-\alpha_{1}^{n, k}\right|\left\|T_{k} U_{n, k-1} y_{n}\right\|+\left|\alpha_{3}^{n+1, k}-\alpha_{3}^{n, k}\right|\left\|y_{n}\right\| \\
& +\left|\alpha_{2}^{n+1, k}-\alpha_{2}^{n, k}\right|\left\|U_{n, k-1} y_{n}\right\| \\
& \leq\left\|U_{n+1, k-1} y_{n}-U_{n, k-1} y_{n}\right\|+\left|\alpha_{1}^{n+1, k}-\alpha_{1}^{n, k}\right|\left\|T_{k} U_{n, k-1} y_{n}\right\| \\
& +\left|\alpha_{3}^{n+1, k}-\alpha_{3}^{n, k}\right|\left\|y_{n}\right\|+\mid 1-\left(\alpha_{1}^{n+1, k}+\alpha_{3}^{n+1, k}\right) \\
& -\left(1-\left(\alpha_{1}^{n, k}+\alpha_{3}^{n, k}\right)\right) \mid\left\|U_{n, k-1} y_{n}\right\| \\
& =\left\|U_{n+1, k-1} y_{n}-U_{n, k-1} y_{n}\right\|\left|\alpha_{1}^{n+1, k}-\alpha_{1}^{n, k}\right|\left\|T_{k} U_{n, k-1} y_{n}\right\| \\
& +\left|\alpha_{3}^{n+1, k}-\alpha_{3}^{n, k}\right|\left\|y_{n}\right\|+\left|\left(\alpha_{1}^{n, k}-\alpha_{1}^{n+1, k}\right)+\left(\alpha_{3}^{n, k}-\alpha_{3}^{n+1, k}\right)\right|\left\|U_{n, k-1} y_{n}\right\| \\
& \leq\left\|U_{n+1, k-1} y_{n}-U_{n, k-1} y_{n}\right\|+\left|\alpha_{1}^{n+1, k}-\alpha_{1}^{n, k}\right|\left\|T_{k} U_{n, k-1} y_{n}\right\| \\
& +\left|\alpha_{3}^{n+1, k}-\alpha_{3}^{n, k}\right|\left\|y_{n}\right\|+\left|\alpha_{1}^{n, k}-\alpha_{1}^{n+1, k}\right|\left\|U_{n, k-1} y_{n}\right\| \\
& +\left|\alpha_{3}^{n, k}-\alpha_{3}^{n+1, k}\right|\left\|U_{n, k-1} y_{n}\right\| \\
& =\left\|U_{n+1, k-1} y_{n}-U_{n, k-1} y_{n}\right\|+\left|\alpha_{1}^{n+1, k}-\alpha_{1}^{n, k}\right|\left(\left\|T_{k} U_{n, k-1} y_{n}\right\|+\left\|U_{n, k-1} y_{n}\right\|\right) \\
& +\left|\alpha_{3}^{n+1, k}-\alpha_{3}^{n, k}\right|\left(\left\|y_{n}\right\|+\left\|U_{n, k-1} y_{n}\right\|\right) \text {. }
\end{aligned}
$$


Fixed Point Theory and Applications

By (3.11), we obtain that for each $n \in \mathbb{N}$,

$$
\begin{aligned}
& \left\|S_{n+1} y_{n}-S_{n} y_{n}\right\|=\left\|U_{n+1, N} y_{n}-U_{n, N} y_{n}\right\| \\
& \leq\left\|U_{n+1, N-1} y_{n}-U_{n, N-1} y_{n}\right\|+\left|\alpha_{1}^{n+1, N}-\alpha_{1}^{n, N}\right|\left(\left\|T_{N} U_{n, N-1} y_{n}\right\|\right. \\
& \left.+\left\|U_{n, N-1} y_{n}\right\|\right)+\left|\alpha_{3}^{n+1, N}-\alpha_{3}^{n, N}\right|\left(\left\|y_{n}\right\|+\left\|U_{n, N-1} y_{n}\right\|\right) \\
& \leq\left\|U_{n+1, N-2} y_{n}-U_{n, N-2} y_{n}\right\|+\left|\alpha_{1}^{n+1, N-1}-\alpha_{1}^{n, N-1}\right| \\
& \times\left(\left\|T_{N-1} U_{n, N-2} y_{n}\right\|+\left\|U_{n, N-2} y_{n}\right\|\right) \\
& +\left|\alpha_{3}^{n+1, N-1}-\alpha_{3}^{n, N-1}\right|\left(\left\|y_{n}\right\|+\left\|U_{n, N-2} y_{n}\right\|\right) \\
& +\left|\alpha_{1}^{n+1, N}-\alpha_{1}^{n, N}\right|\left(\left\|T_{N} U_{n, N-1} y_{n}\right\|+\left\|U_{n, N-1} y_{n}\right\|\right) \\
& +\left|\alpha_{3}^{n+1, N}-\alpha_{3}^{n, N}\right|\left(\left\|y_{n}\right\|+\left\|U_{n, N-1} y_{n}\right\|\right) \\
& =\left\|U_{n+1, N-2} y_{n}-U_{n, N-2} y_{n}\right\|+\sum_{j=N-1}^{N}\left|\alpha_{1}^{n+1, j}-\alpha_{1}^{n, j}\right|\left(\left\|T_{j} U_{n, j-1} y_{n}\right\|\right. \\
& \left.+\left\|U_{n, j-1} y_{n}\right\|\right)+\sum_{j=N-1}^{N}\left|\alpha_{3}^{n+1, j}-\alpha_{3}^{n, j}\right|\left(\left\|y_{n}\right\|+\left\|U_{n, j-1} y_{n}\right\|\right) \\
& \leq \\
& \vdots \\
& \leq\left\|U_{n+1,1} y_{n}-U_{n, 1} y_{n}\right\|+\sum_{j=2}^{N}\left|\alpha_{1}^{n+1, j}-\alpha_{1}^{n, j}\right|\left(\left\|T_{j} U_{n, j-1} y_{n}\right\|+\left\|U_{n, j-1} y_{n}\right\|\right) \\
& +\sum_{j=2}^{N}\left|\alpha_{3}^{n+1, j}-\alpha_{3}^{n, j}\right|\left(\left\|y_{n}\right\|+\left\|U_{n, j-1} y_{n}\right\|\right) \\
& =\left\|\left(1-\alpha_{1}^{n+1,1}\right) y_{n}+\alpha_{1}^{n+1,1} T_{1} y_{n}-\left(1-\alpha_{1}^{n, 1}\right) y_{n}-\alpha_{1}^{n, 1} T_{1} y_{n}\right\| \\
& +\sum_{j=2}^{N}\left|\alpha_{1}^{n+1, j}-\alpha_{1}^{n, j}\right|\left(\left\|T_{j} U_{n, j-1} y_{n}\right\|+\left\|U_{n, j-1} y_{n}\right\|\right) \\
& +\sum_{j=2}^{N}\left|\alpha_{3}^{n+1, j}-\alpha_{3}^{n, j}\right|\left(\left\|y_{n}\right\|+\left\|U_{n, j-1} y_{n}\right\|\right) \\
& =\left|\alpha_{1}^{n+1,1}-\alpha_{1}^{n, 1}\right|\left\|T_{1} y_{n}-y_{n}\right\| \\
& +\sum_{j=2}^{N}\left|\alpha_{1}^{n+1, j}-\alpha_{1}^{n, j}\right|\left(\left\|T_{j} U_{n, j-1} y_{n}\right\|+\left\|U_{n, j-1} y_{n}\right\|\right) \\
& +\sum_{j=2}^{N}\left|\alpha_{3}^{n+1, j}-\alpha_{3}^{n, j}\right|\left(\left\|y_{n}\right\|+\left\|U_{n, j-1} y_{n}\right\|\right) .
\end{aligned}
$$


This together with the condition (iv), we obtain

$$
\lim _{n \rightarrow \infty}\left\|S_{n+1} y_{n}-S_{n} y_{n}\right\|=0
$$

By (3.10), (3.13) and conditions (i), (ii), (iii), (iv), it implies that

$$
\limsup _{n \rightarrow \infty}\left(\left\|k_{n+1}-k_{n}\right\|-\left\|x_{n+1}-x_{n}\right\|\right) \leq 0 .
$$

From Lemma 2.5, (3.9), (3.14) and condition (ii), we have

$$
\lim _{n \rightarrow \infty}\left\|x_{n}-k_{n}\right\|=0
$$

From (3.9), we can rewrite

$$
x_{n+1}-x_{n}=\left(1-\beta_{n}\right)\left(k_{n}-x_{n}\right) .
$$

By (3.15), we have

$$
\lim _{n \rightarrow \infty}\left\|x_{n+1}-x_{n}\right\|=0
$$

On the other hand, we have

$$
\begin{aligned}
\left\|x_{n}-S_{n} y_{n}\right\| & \leq\left\|x_{n}-x_{n+1}\right\|+\left\|x_{n+1}-S_{n} y_{n}\right\| \\
& =\left\|x_{n}-x_{n+1}\right\|+\left\|\alpha_{n} f\left(x_{n}\right)+\beta_{n} x_{n}+\gamma_{n} S_{n} y_{n}-S_{n} y_{n}\right\| \\
& =\left\|x_{n}-x_{n+1}\right\|+\left\|\alpha_{n}\left(f\left(x_{n}\right)-S_{n} y_{n}\right)+\beta_{n}\left(x_{n}-S_{n} y_{n}\right)\right\| \\
& =\left\|x_{n}-x_{n+1}\right\|+\alpha_{n}\left\|f\left(x_{n}\right)-S_{n} y_{n}\right\|+\beta_{n}\left\|x_{n}-S_{n} y_{n}\right\| .
\end{aligned}
$$

This implies that

$$
\left(1-\beta_{n}\right)\left\|x_{n}-S_{n} y_{n}\right\| \leq\left\|x_{n}-x_{n+1}\right\|+\alpha_{n}\left\|f\left(x_{n}\right)-S_{n} y_{n}\right\|
$$

By (3.17) and condition (ii), we have

$$
\lim _{n \rightarrow \infty}\left\|x_{n}-S_{n} y_{n}\right\|=0
$$

Step 3. Let $z \in \mathfrak{F}$; we show that

$$
\lim _{n \rightarrow \infty}\left\|A u_{n}-A z\right\|=\lim _{n \rightarrow \infty}\left\|B v_{n}-B z\right\|=\lim _{n \rightarrow \infty}\left\|A x_{n}-A z\right\|=\lim _{n \rightarrow \infty}\left\|B x_{n}-B z\right\|=0
$$


From definition of $y_{n}$, we have

$$
\begin{aligned}
& \left\|y_{n}-z\right\|^{2}=\| \delta_{n}\left(P_{C}\left(u_{n}-\lambda_{n} A u_{n}\right)-P_{C}\left(I-\lambda_{n} A\right) z\right)+\left(1-\delta_{n}\right)\left(P_{C}\left(v_{n}-\eta_{n} B v_{n}\right)\right. \\
& \left.-P_{C}\left(I-\eta_{n} B\right) z\right) \|^{2} \\
& \leq \delta_{n}\left\|\left(P_{C}\left(u_{n}-\lambda_{n} A u_{n}\right)-P_{C}\left(I-\lambda_{n} A\right) z\right)\right\|^{2} \\
& +\left(1-\delta_{n}\right)\left\|\left(P_{C}\left(v_{n}-\eta_{n} B v_{n}\right)-P_{C}\left(I-\eta_{n} B\right) z\right)\right\|^{2} \\
& \leq \delta_{n}\left\|u_{n}-\lambda_{n} A u_{n}-z+\lambda_{n} A z\right\|^{2}+\left(1-\delta_{n}\right)\left\|v_{n}-\eta_{n} B v_{n}-z+\eta_{n} B z\right\|^{2} \\
& =\delta_{n}\left\|\left(u_{n}-z\right)-\lambda_{n}\left(A u_{n}-A z\right)\right\|^{2}+\left(1-\delta_{n}\right)\left\|\left(v_{n}-z\right)-\eta_{n}\left(B v_{n}-B z\right)\right\|^{2} \\
& =\delta_{n}\left(\left\|u_{n}-z\right\|^{2}+\lambda_{n}^{2}\left\|\left(A u_{n}-A z\right)\right\|^{2}-2 \lambda_{n}\left\langle u_{n}-z, A u_{n}-A z\right\rangle\right) \\
& +\left(1-\delta_{n}\right)\left(\left\|v_{n}-z\right\|^{2}+\eta_{n}^{2}\left\|B v_{n}-B z\right\|^{2}-2 \eta_{n}\left\langle v_{n}-z, B v_{n}-B z\right\rangle\right) \\
& \leq \delta_{n}\left(\left\|u_{n}-z\right\|^{2}+\lambda_{n}^{2}\left\|\left(A u_{n}-A z\right)\right\|^{2}-2 \lambda_{n} \alpha\left\|A u_{n}-A z\right\|^{2}\right) \\
& +\left(1-\delta_{n}\right)\left(\left\|v_{n}-z\right\|^{2}+\eta_{n}^{2}\left\|B v_{n}-B z\right\|^{2}-2 \eta_{n} \beta\left\|B v_{n}-B z\right\|^{2}\right) \\
& =\delta_{n}\left(\left\|u_{n}-z\right\|^{2}-\lambda_{n}\left(2 \alpha-\lambda_{n}\right)\left\|\left(A u_{n}-A z\right)\right\|^{2}\right) \\
& +\left(1-\delta_{n}\right)\left(\left\|v_{n}-z\right\|^{2}-\eta_{n}\left(2 \beta-\eta_{n}\right)\left\|B v_{n}-B z\right\|^{2}\right) \\
& =\delta_{n}\left(\left\|T_{r_{n}}\left(I-r_{n} A\right) x_{n}-T_{r_{n}}\left(z-r_{n} A z\right)\right\|^{2}-\lambda_{n}\left(2 \alpha-\lambda_{n}\right)\left\|\left(A u_{n}-A z\right)\right\|^{2}\right) \\
& +\left(1-\delta_{n}\right)\left(\left\|T_{S_{n}}\left(I-s_{n} B\right) x_{n}-T_{s_{n}}\left(z-s_{n} B z\right)\right\|^{2}-\eta_{n}\left(2 \beta-\eta_{n}\right)\left\|B v_{n}-B z\right\|^{2}\right) \\
& \leq \delta_{n}\left(\left\|x_{n}-z\right\|^{2}-\lambda_{n}\left(2 \alpha-\lambda_{n}\right)\left\|\left(A u_{n}-A z\right)\right\|^{2}\right) \\
& +\left(1-\delta_{n}\right)\left(\left\|x_{n}-z\right\|^{2}-\eta_{n}\left(2 \beta-\eta_{n}\right)\left\|B v_{n}-B z\right\|^{2}\right) \\
& =\left\|x_{n}-z\right\|^{2}-\lambda_{n} \delta_{n}\left(2 \alpha-\lambda_{n}\right)\left\|\left(A u_{n}-A z\right)\right\|^{2} \\
& -\eta_{n}\left(1-\delta_{n}\right)\left(2 \beta-\eta_{n}\right)\left\|B v_{n}-B z\right\|^{2} .
\end{aligned}
$$


By (3.23), we have

$$
\begin{aligned}
\left\|x_{n+1}-z\right\|^{2}= & \left\|\alpha_{n}\left(f\left(x_{n}\right)-z\right)+\beta_{n}\left(x_{n}-z\right)+\gamma_{n}\left(S_{n} y_{n}-z\right)\right\|^{2} \\
\leq & \alpha_{n}\left\|f\left(x_{n}\right)-z\right\|^{2}+\beta_{n}\left\|x_{n}-z\right\|^{2}+\gamma_{n}\left\|S_{n} y_{n}-z\right\|^{2} \\
\leq & \alpha_{n}\left\|f\left(x_{n}\right)-z\right\|^{2}+\beta_{n}\left\|x_{n}-z\right\|^{2}+\gamma_{n}\left\|y_{n}-z\right\|^{2} \\
\leq & \alpha_{n}\left\|f\left(x_{n}\right)-z\right\|^{2}+\beta_{n}\left\|x_{n}-z\right\|^{2}+\gamma_{n}\left(\left\|x_{n}-z\right\|^{2}-\lambda_{n} \delta_{n}\left(2 \alpha-\lambda_{n}\right)\left\|\left(A u_{n}-A z\right)\right\|^{2}\right. \\
& \left.-\eta_{n}\left(1-\delta_{n}\right)\left(2 \beta-\eta_{n}\right)\left\|B v_{n}-B z\right\|^{2}\right) \\
= & \alpha_{n}\left\|f\left(x_{n}\right)-z\right\|^{2}+\beta_{n}\left\|x_{n}-z\right\|^{2}+\gamma_{n}\left\|x_{n}-z\right\|^{2}-\lambda_{n} \gamma_{n} \delta_{n}\left(2 \alpha-\lambda_{n}\right)\left\|\left(A u_{n}-A z\right)\right\|^{2} \\
& -\eta_{n} \gamma_{n}\left(1-\delta_{n}\right)\left(2 \beta-\eta_{n}\right)\left\|B v_{n}-B z\right\|^{2} \\
\leq & \alpha_{n}\left\|f\left(x_{n}\right)-z\right\|^{2}+\left\|x_{n}-z\right\|^{2}-\lambda_{n} \gamma_{n} \delta_{n}\left(2 \alpha-\lambda_{n}\right)\left\|\left(A u_{n}-A z\right)\right\|^{2} \\
& -\eta_{n} \gamma_{n}\left(1-\delta_{n}\right)\left(2 \beta-\eta_{n}\right)\left\|B v_{n}-B z\right\|^{2} .
\end{aligned}
$$

By (3.24), we have

$$
\begin{aligned}
\lambda_{n} \gamma_{n} \delta_{n}\left(2 \alpha-\lambda_{n}\right)\left\|\left(A u_{n}-A z\right)\right\|^{2} \leq & \alpha_{n}\left\|f\left(x_{n}\right)-z\right\|^{2}+\left\|x_{n}-z\right\|^{2}-\left\|x_{n+1}-z\right\|^{2} \\
& -\eta_{n} \gamma_{n}\left(1-\delta_{n}\right)\left(2 \beta-\eta_{n}\right)\left\|B v_{n}-B z\right\|^{2} \\
\leq & \alpha_{n}\left\|f\left(x_{n}\right)-z\right\|^{2}+\left(\left\|x_{n}-z\right\|+\left\|x_{n+1}-z\right\|\right)\left\|x_{n+1}-x_{n}\right\| .
\end{aligned}
$$

From conditions (i)-(iii) and (3.17), we have

$$
\lim _{n \rightarrow \infty}\left\|A u_{n}-A z\right\|^{2}=0 .
$$

By using the same method as (3.26), we have

$$
\lim _{n \rightarrow \infty}\left\|B v_{n}-B z\right\|^{2}=0 .
$$


By nonexpansiveness of $T_{r_{n}}, T_{S_{n}}, I-\lambda_{n} A, I-\eta_{n} B$ and (3.23), we have

$$
\begin{aligned}
\left\|y_{n}-z\right\|^{2} \leq & \delta_{n}\left\|\left(P_{C}\left(u_{n}-\lambda_{n} A u_{n}\right)-P_{C}\left(I-\lambda_{n} A\right) z\right)\right\|^{2} \\
& +\left(1-\delta_{n}\right)\left\|P_{C}\left(v_{n}-\eta_{n} B v_{n}\right)-P_{C}\left(I-\eta_{n} B\right) z\right\|^{2} \\
\leq & \delta_{n}\left\|\left(I-\lambda_{n} A\right) u_{n}-\left(I-\lambda_{n} A\right) z\right\|^{2}+\left(1-\delta_{n}\right)\left\|\left(I-\eta_{n} B\right) v_{n}-\left(I-\eta_{n} B\right) z\right\|^{2} \\
\leq & \delta_{n}\left\|u_{n}-z\right\|^{2}+\left(1-\delta_{n}\right)\left\|v_{n}-z\right\|^{2} \\
= & \delta_{n}\left\|T_{r_{n}}\left(I-r_{n} A\right) x_{n}-T_{r_{n}}\left(I-r_{n} A\right) z\right\|^{2}+\left(1-\delta_{n}\right) \| T_{s_{n}}\left(I-s_{n} B\right) x_{n} \\
- & T_{s_{n}}\left(I-s_{n} B\right) z \|^{2} \\
\leq & \delta_{n}\left\|\left(I-r_{n} A\right) x_{n}-\left(I-r_{n} A\right) z\right\|^{2}+\left(1-\delta_{n}\right)\left\|\left(I-s_{n} B\right) x_{n}-\left(I-s_{n} B\right) z\right\|^{2} \\
= & \delta_{n}\left\|x_{n}-r_{n} A x_{n}-z+r_{n} A z\right\|^{2}+\left(1-\delta_{n}\right)\left\|x_{n}-s_{n} B x_{n}-z+s_{n} B z\right\|^{2} \\
= & \delta_{n}\left\|\left(x_{n}-z\right)-r_{n}\left(A x_{n}-A z\right)\right\|^{2}+\left(1-\delta_{n}\right)\left\|\left(x_{n}-z\right)-s_{n}\left(B x_{n}-B z\right)\right\|^{2} \\
= & \delta_{n}\left(\left\|x_{n}-z\right\|^{2}+r_{n}^{2}\left\|A x_{n}-A z\right\|^{2}-2 r_{n}\left\langle x_{n}-z, A x_{n}-A z\right\rangle\right) \\
& +\left(1-\delta_{n}\right)\left(\left\|x_{n}-z\right\|^{2}+s_{n}^{2}\left\|B x_{n}-B z\right\|^{2}-2 s_{n}\left\langle x_{n}-z B x_{n}-B z\right\rangle\right) \\
= & \delta_{n}\left\|x_{n}-z\right\|^{2}+r_{n}^{2} \delta_{n}\left\|A x_{n}-A z\right\|^{2}-2 \delta_{n} r_{n}\left\langle x_{n}-z, A x_{n}-A z\right\rangle \\
& +\left(1-\delta_{n}\right)\left\|x_{n}-z\right\|^{2}+s_{n}^{2}\left(1-\delta_{n}\right)\left\|B x_{n}-B z\right\|^{2}-2 s_{n}\left(1-\delta_{n}\right)\left\langle x_{n}-z B x_{n}-B z\right\rangle \\
\leq & \left\|x_{n}-z\right\|^{2}+r_{n}^{2} \delta_{n}\left\|A x_{n}-A z\right\|^{2}-2 \delta_{n} r_{n} \alpha\left\|A x_{n}-A z\right\|^{2} \\
& +s_{n}^{2}\left(1-\delta_{n}\right)\left\|B x_{n}-B z\right\|^{2}-2 s_{n}\left(1-\delta_{n}\right) \beta\left\|B x_{n}-B z\right\|^{2} \\
= & \left\|x_{n}-z\right\|^{2}-\delta_{n} r_{n}\left(2 \alpha-r_{n}\right)\left\|A x_{n}-A z\right\|^{2}-s_{n}\left(1-\delta_{n}\right)\left(2 \beta-s_{n}\right)\left\|B x_{n}-B z\right\|^{2} .
\end{aligned}
$$

By (3.28), we have

$$
\begin{aligned}
\left\|x_{n+1}-z\right\|^{2}= & \left\|\alpha_{n}\left(f\left(x_{n}\right)-z\right)+\beta_{n}\left(x_{n}-z\right)+\gamma_{n}\left(S_{n} y_{n}-z\right)\right\|^{2} \\
\leq & \alpha_{n}\left\|f\left(x_{n}\right)-z\right\|^{2}+\beta_{n}\left\|x_{n}-z\right\|^{2}+\gamma_{n}\left\|S_{n} y_{n}-z\right\|^{2} \\
\leq & \alpha_{n}\left\|f\left(x_{n}\right)-z\right\|^{2}+\beta_{n}\left\|x_{n}-z\right\|^{2}+\gamma_{n}\left\|y_{n}-z\right\|^{2} \\
\leq & \alpha_{n}\left\|f\left(x_{n}\right)-z\right\|^{2}+\beta_{n}\left\|x_{n}-z\right\|^{2} \\
& +\gamma_{n}\left(\left\|x_{n}-z\right\|^{2}-\delta_{n} r_{n}\left(2 \alpha-r_{n}\right)\left\|A x_{n}-A z\right\|^{2}\right. \\
& \left.\quad-s_{n}\left(1-\delta_{n}\right)\left(2 \beta-S_{n}\right)\left\|B x_{n}-B z\right\|^{2}\right) \\
& \quad \alpha_{n}\left\|f\left(x_{n}\right)-z\right\|^{2}+\beta_{n}\left\|x_{n}-z\right\|^{2}+\gamma_{n}\left\|x_{n}-z\right\|^{2}-\delta_{n} \gamma_{n} r_{n}\left(2 \alpha-r_{n}\right)\left\|A x_{n}-A z\right\|^{2}
\end{aligned}
$$




$$
\begin{aligned}
& -s_{n} \gamma_{n}\left(1-\delta_{n}\right)\left(2 \beta-s_{n}\right)\left\|B x_{n}-B z\right\|^{2} \\
\leq & \alpha_{n}\left\|f\left(x_{n}\right)-z\right\|^{2}+\left\|x_{n}-z\right\|^{2}-\delta_{n} \gamma_{n} r_{n}\left(2 \alpha-r_{n}\right)\left\|A x_{n}-A z\right\|^{2} \\
& -s_{n} \gamma_{n}\left(1-\delta_{n}\right)\left(2 \beta-s_{n}\right)\left\|B x_{n}-B z\right\|^{2} .
\end{aligned}
$$

By (3.29), we have

$$
\begin{aligned}
\delta_{n} \gamma_{n} r_{n}\left(2 \alpha-r_{n}\right)\left\|A x_{n}-A z\right\|^{2} \leq & \alpha_{n}\left\|f\left(x_{n}\right)-z\right\|^{2}+\left\|x_{n}-z\right\|^{2}-\left\|x_{n+1}-z\right\|^{2} \\
& -s_{n} \gamma_{n}\left(1-\delta_{n}\right)\left(2 \beta-s_{n}\right)\left\|B x_{n}-B z\right\|^{2} \\
\leq & \alpha_{n}\left\|f\left(x_{n}\right)-z\right\|^{2}+\left(\left\|x_{n}-z\right\|+\left\|x_{n+1}-z\right\|\right)\left\|x_{n+1}-x_{n}\right\| .
\end{aligned}
$$

From (3.17) and conditions (i)-(iii), we have

$$
\lim _{n \rightarrow \infty}\left\|A x_{n}-A z\right\|=0
$$

By using the same method as (3.31), we have

$$
\lim _{n \rightarrow \infty}\left\|B x_{n}-B z\right\|=0
$$

Step 4. We will show that

$$
\lim _{n \rightarrow \infty}\left\|y_{n}-x_{n}\right\|=0
$$

Putting $M_{n}=P_{C}\left(u_{n}-\lambda_{n} A u_{n}\right)$ and $N_{n}=P_{C}\left(v_{n}-\eta_{n} B v_{n}\right)$, we will show that

$$
\lim _{n \rightarrow \infty}\left\|u_{n}-x_{n}\right\|=\lim _{n \rightarrow \infty}\left\|v_{n}-x_{n}\right\|=\lim _{n \rightarrow \infty}\left\|M_{n}-u_{n}\right\|=\lim _{n \rightarrow \infty}\left\|N_{n}-v_{n}\right\|=0 .
$$

Let $z \in \mathfrak{F}$; by (3.28), we have

$$
\begin{aligned}
\left\|y_{n}-z\right\|^{2} & \leq \delta_{n}\left\|M_{n}-z\right\|^{2}+\left(1-\delta_{n}\right)\left\|N_{n}-z\right\|^{2} \\
& \leq \delta_{n}\left\|u_{n}-z\right\|^{2}+\left(1-\delta_{n}\right)\left\|v_{n}-z\right\|^{2} .
\end{aligned}
$$


By nonexpansiveness of $I-r_{n} A$, we have

$$
\begin{aligned}
\left\|u_{n}-z\right\|^{2}= & \left\|T_{r_{n}}\left(x_{n}-r_{n} A x_{n}\right)-T_{r_{n}}\left(z-r_{n} A z\right)\right\|^{2} \\
\leq & \left\langle\left(x_{n}-r_{n} A x_{n}\right)-\left(z-r_{n} A z\right), u_{n}-z\right\rangle \\
= & \frac{1}{2}\left(\left\|\left(x_{n}-r_{n} A x_{n}\right)-\left(z-r_{n} A z\right)\right\|^{2}+\left\|u_{n}-z\right\|^{2}\right. \\
& \left.-\left\|\left(x_{n}-r_{n} A x_{n}\right)-\left(z-r_{n} A z\right)-\left(u_{n}-z\right)\right\|^{2}\right) \\
\leq & \frac{1}{2}\left(\left\|x_{n}-z\right\|^{2}+\left\|u_{n}-z\right\|^{2}-\left\|\left(x_{n}-u_{n}\right)-r_{n}\left(A x_{n}-A z\right)\right\|^{2}\right) \\
= & \frac{1}{2}\left(\left\|x_{n}-z\right\|^{2}+\left\|u_{n}-z\right\|^{2}-\left\|x_{n}-u_{n}\right\|^{2}\right. \\
& \left.+2 r_{n}\left\langle x_{n}-u_{n}, A x_{n}-A z\right\rangle-r_{n}^{2}\left\|A x_{n}-A z\right\|^{2}\right) .
\end{aligned}
$$

This implies

$$
\left\|u_{n}-z\right\|^{2} \leq\left\|x_{n}-z\right\|^{2}-\left\|x_{n}-u_{n}\right\|^{2}+2 r_{n}\left\langle x_{n}-u_{n}, A x_{n}-A z\right\rangle-r_{n}^{2}\left\|A x_{n}-A z\right\|^{2} .
$$

By using the same method as (3.37), we have

$$
\left\|v_{n}-z\right\|^{2} \leq\left\|x_{n}-z\right\|^{2}-\left\|x_{n}-v_{n}\right\|^{2}+2 s_{n}\left\langle x_{n}-v_{n}, B x_{n}-B z\right\rangle-s_{n}^{2}\left\|B x_{n}-B z\right\|^{2} .
$$

Substituting (3.37) and (3.38) into (3.35), we have

$$
\begin{aligned}
\left\|y_{n}-z\right\|^{2} \leq & \delta_{n}\left\|u_{n}-z\right\|^{2}+\left(1-\delta_{n}\right)\left\|v_{n}-z\right\|^{2} \\
\leq & \delta_{n}\left(\left\|x_{n}-z\right\|^{2}-\left\|x_{n}-u_{n}\right\|^{2}+2 r_{n}\left\langle x_{n}-u_{n}, A x_{n}-A z\right\rangle-r_{n}^{2}\left\|A x_{n}-A z\right\|^{2}\right) \\
& +\left(1-\delta_{n}\right)\left(\left\|x_{n}-z\right\|^{2}-\left\|x_{n}-v_{n}\right\|^{2}+2 s_{n}\left\langle x_{n}-v_{n}, B x_{n}-B z\right\rangle-s_{n}^{2}\left\|B x_{n}-B z\right\|^{2}\right) \\
\leq & \delta_{n}\left\|x_{n}-z\right\|^{2}-\delta_{n}\left\|x_{n}-u_{n}\right\|^{2}+2 \delta_{n} r_{n}\left\|x_{n}-u_{n}\right\|\left\|A x_{n}-A z\right\|+\left(1-\delta_{n}\right)\left\|x_{n}-z\right\|^{2} \\
& -\left(1-\delta_{n}\right)\left\|x_{n}-v_{n}\right\|^{2}+2 s_{n}\left(1-\delta_{n}\right)\left\|x_{n}-v_{n}\right\|\left\|B x_{n}-B z\right\| \\
= & \left\|x_{n}-z\right\|^{2}-\delta_{n}\left\|x_{n}-u_{n}\right\|^{2}+2 \delta_{n} r_{n}\left\|x_{n}-u_{n}\right\|\left\|A x_{n}-A z\right\|-\left(1-\delta_{n}\right)\left\|x_{n}-v_{n}\right\|^{2} \\
& +2 s_{n}\left(1-\delta_{n}\right)\left\|x_{n}-v_{n}\right\|\left\|B x_{n}-B z\right\| .
\end{aligned}
$$


By (3.39), we have

$$
\begin{aligned}
\left\|x_{n+1}-z\right\|^{2} \leq & \alpha_{n}\left\|f\left(x_{n}\right)-z\right\|^{2}+\beta_{n}\left\|x_{n}-z\right\|^{2}+\gamma_{n}\left\|y_{n}-z\right\|^{2} \\
\leq & \alpha_{n}\left\|f\left(x_{n}\right)-z\right\|^{2}+\beta_{n}\left\|x_{n}-z\right\|^{2} \\
+ & \gamma_{n}\left(\left\|x_{n}-z\right\|^{2}-\delta_{n}\left\|x_{n}-u_{n}\right\|^{2}\right. \\
& +2 \delta_{n} r_{n}\left\|x_{n}-u_{n}\right\|\left\|A x_{n}-A z\right\|-\left(1-\delta_{n}\right)\left\|x_{n}-v_{n}\right\|^{2} \\
& \left.+2 s_{n}\left(1-\delta_{n}\right)\left\|x_{n}-v_{n}\right\|\left\|B x_{n}-B z\right\|\right) \\
= & \alpha_{n}\left\|f\left(x_{n}\right)-z\right\|^{2}+\beta_{n}\left\|x_{n}-z\right\|^{2}+\gamma_{n}\left\|x_{n}-z\right\|^{2}-\gamma_{n} \delta_{n}\left\|x_{n}-u_{n}\right\|^{2} \\
& +2 \gamma_{n} \delta_{n} r_{n}\left\|x_{n}-u_{n}\right\|\left\|A x_{n}-A z\right\|-\left(1-\delta_{n}\right) \gamma_{n}\left\|x_{n}-v_{n}\right\|^{2} \\
& +2 s_{n} \gamma_{n}\left(1-\delta_{n}\right)\left\|x_{n}-v_{n}\right\|\left\|B x_{n}-B z\right\| \\
\leq & \alpha_{n}\left\|f\left(x_{n}\right)-z\right\|^{2}+\left\|x_{n}-z\right\|^{2}-\gamma_{n} \delta_{n}\left\|x_{n}-u_{n}\right\|^{2} \\
& +2 \gamma_{n} \delta_{n} r_{n}\left\|x_{n}-u_{n}\right\|\left\|A x_{n}-A z\right\|-\left(1-\delta_{n}\right) \gamma_{n}\left\|x_{n}-v_{n}\right\|^{2} \\
& +2 s_{n} \gamma_{n}\left(1-\delta_{n}\right)\left\|x_{n}-v_{n}\right\|\left\|B x_{n}-B z\right\| .
\end{aligned}
$$

It follows that

$$
\begin{aligned}
\gamma_{n} \delta_{n}\left\|x_{n}-u_{n}\right\|^{2} \leq & \alpha_{n}\left\|f\left(x_{n}\right)-z\right\|^{2}+\left\|x_{n}-z\right\|^{2}-\left\|x_{n+1}-z\right\|^{2} \\
& +2 \gamma_{n} \delta_{n} r_{n}\left\|x_{n}-u_{n}\right\|\left\|A x_{n}-A z\right\|-\left(1-\delta_{n}\right) \gamma_{n}\left\|x_{n}-v_{n}\right\|^{2} \\
& +2 s_{n} \gamma_{n}\left(1-\delta_{n}\right)\left\|x_{n}-v_{n}\right\|\left\|B x_{n}-B z\right\| \\
\leq & \alpha_{n}\left\|f\left(x_{n}\right)-z\right\|^{2}+\left(\left\|x_{n}-z\right\|+\left\|x_{n+1}-z\right\|\right)\left\|x_{n+1}-x_{n}\right\| \\
& +2 \gamma_{n} \delta_{n} r_{n}\left\|x_{n}-u_{n}\right\|\left\|A x_{n}-A z\right\|+2 s_{n} \gamma_{n}\left(1-\delta_{n}\right)\left\|x_{n}-v_{n}\right\|\left\|B x_{n}-B z\right\| .
\end{aligned}
$$

By conditions (i)-(iii), (3.41), (3.31), (3.32), and (3.17), we have

$$
\lim _{n \rightarrow \infty}\left\|x_{n}-u_{n}\right\|=0
$$

By using the same method as (3.42), we have

$$
\lim _{n \rightarrow \infty}\left\|x_{n}-v_{n}\right\|=0
$$


Fixed Point Theory and Applications

By nonexpansiveness of $T_{r_{n}}\left(I-r_{n} A\right)$, we have

$$
\begin{aligned}
\left\|M_{n}-z\right\|^{2}= & \left\|P_{C}\left(u_{n}-\lambda_{n} A u_{n}\right)-P_{C}\left(z-\lambda_{n} A z\right)\right\|^{2} \\
\leq & \left\langle\left(u_{n}-\alpha_{n} A u_{n}\right)-\left(z-\alpha_{n} A z\right), M_{n}-z\right\rangle \\
= & \frac{1}{2}\left(\left\|\left(u_{n}-\alpha_{n} A u_{n}\right)-\left(z-\alpha_{n} A z\right)\right\|^{2}+\left\|M_{n}-z\right\|^{2}-\|\left(u_{n}-\alpha_{n} A u_{n}\right)\right. \\
& \left.\quad-\left(z-\alpha_{n} A z\right)-\left(M_{n}-z\right) \|^{2}\right) \\
\leq & \frac{1}{2}\left(\left\|u_{n}-z\right\|^{2}+\left\|M_{n}-z\right\|^{2}-\left\|\left(u_{n}-M_{n}\right)-\alpha_{n}\left(A u_{n}-A z\right)\right\|^{2}\right) \\
= & \frac{1}{2}\left(\left\|T_{r_{n}}\left(I-r_{n} A\right) x_{n}-T_{r_{n}}\left(I-r_{n} A\right) z\right\|^{2}+\left\|M_{n}-z\right\|^{2}-\left\|u_{n}-M_{n}\right\|^{2}\right. \\
& \left.\quad+2 \alpha_{n}\left\langle u_{n}-M_{n}, A u_{n}-A z\right\rangle-\alpha_{n}^{2}\left\|A u_{n}-A z\right\|^{2}\right) \\
\leq & \frac{1}{2}\left(\left\|x_{n}-z\right\|^{2}+\left\|M_{n}-z\right\|^{2}-\left\|u_{n}-M_{n}\right\|^{2}+2 \alpha_{n}\left\langle u_{n}-M_{n}, A u_{n}-A z\right\rangle\right. \\
& \left.\quad-\alpha_{n}^{2}\left\|A u_{n}-A z\right\|^{2}\right) .
\end{aligned}
$$

Hence, we have

$$
\begin{aligned}
\left\|M_{n}-z\right\|^{2} \leq & \left\|x_{n}-z\right\|^{2}-\left\|u_{n}-M_{n}\right\|^{2}+2 \alpha_{n}\left\langle u_{n}-M_{n}, A u_{n}-A z\right\rangle \\
& -\alpha_{n}^{2}\left\|A u_{n}-A z\right\|^{2} .
\end{aligned}
$$

By using the same method as (3.45), we have

$$
\left\|N_{n}-z\right\|^{2} \leq\left\|x_{n}-z\right\|^{2}-\left\|v_{n}-N_{n}\right\|^{2}+2 \eta_{n}\left\langle v_{n}-N_{n}, B v_{n}-B z\right\rangle-\eta_{n}^{2}\left\|B v_{n}-B z\right\|^{2} .
$$

Substituting (3.45) and (3.46) into (3.35), we have

$$
\begin{aligned}
\left\|y_{n}-z\right\|^{2} \leq & \delta_{n}\left\|M_{n}-z\right\|^{2}+\left(1-\delta_{n}\right)\left\|N_{n}-z\right\|^{2} \\
\leq & \delta_{n}\left(\left\|x_{n}-z\right\|^{2}-\left\|u_{n}-M_{n}\right\|^{2}+2 \alpha_{n}\left\langle u_{n}-M_{n}, A u_{n}-A z\right\rangle-\alpha_{n}^{2}\left\|A u_{n}-A z\right\|^{2}\right) \\
& +\left(1-\delta_{n}\right)\left(\left\|x_{n}-z\right\|^{2}-\left\|v_{n}-N_{n}\right\|^{2}+2 \eta_{n}\left\langle v_{n}-N_{n}, B v_{n}-B z\right\rangle-\eta_{n}^{2}\left\|B v_{n}-B z\right\|^{2}\right) \\
\leq & \delta_{n}\left\|x_{n}-z\right\|^{2}-\delta_{n}\left\|u_{n}-M_{n}\right\|^{2}+2 \delta_{n} \alpha_{n}\left\|u_{n}-M_{n}\right\|\left\|A u_{n}-A z\right\| \\
& +\left(1-\delta_{n}\right)\left\|x_{n}-z\right\|^{2}-\left(1-\delta_{n}\right)\left\|v_{n}-N_{n}\right\|^{2}+2\left(1-\delta_{n}\right) \eta_{n}\left\|v_{n}-N_{n}\right\|\left\|B v_{n}-B z\right\| \\
= & \left\|x_{n}-z\right\|^{2}-\delta_{n}\left\|u_{n}-M_{n}\right\|^{2}+2 \delta_{n} \alpha_{n}\left\|u_{n}-M_{n}\right\|\left\|A u_{n}-A z\right\|-\left(1-\delta_{n}\right)\left\|v_{n}-N_{n}\right\|^{2} \\
& +2\left(1-\delta_{n}\right) \eta_{n}\left\|v_{n}-N_{n}\right\|\left\|B v_{n}-B z\right\| .
\end{aligned}
$$


By (3.47), we have

$$
\begin{aligned}
\left\|x_{n+1}-z\right\|^{2} \leq & \alpha_{n}\left\|f\left(x_{n}\right)-z\right\|^{2}+\beta_{n}\left\|x_{n}-z\right\|^{2}+\gamma_{n}\left\|y_{n}-z\right\|^{2} \\
\leq & \alpha_{n}\left\|f\left(x_{n}\right)-z\right\|^{2}+\beta_{n}\left\|x_{n}-z\right\|^{2} \\
& +\gamma_{n}\left(\left\|x_{n}-z\right\|^{2}-\delta_{n}\left\|u_{n}-M_{n}\right\|^{2}\right. \\
& +2 \delta_{n} \alpha_{n}\left\|u_{n}-M_{n}\right\|\left\|A u_{n}-A z\right\|-\left(1-\delta_{n}\right)\left\|v_{n}-N_{n}\right\|^{2} \\
& \left.+2\left(1-\delta_{n}\right) \eta_{n}\left\|v_{n}-N_{n}\right\|\left\|B v_{n}-B z\right\|\right) \\
= & \alpha_{n}\left\|f\left(x_{n}\right)-z\right\|^{2}+\beta_{n}\left\|x_{n}-z\right\|^{2}+\gamma_{n}\left\|x_{n}-z\right\|^{2}-\delta_{n} \gamma_{n}\left\|u_{n}-M_{n}\right\|^{2} \\
& +2 \delta_{n} \gamma_{n} \alpha_{n}\left\|u_{n}-M_{n}\right\|\left\|A u_{n}-A z\right\|-\left(1-\delta_{n}\right) \gamma_{n}\left\|v_{n}-N_{n}\right\|^{2} \\
& +2\left(1-\delta_{n}\right) \gamma_{n} \eta_{n}\left\|v_{n}-N_{n}\right\|\left\|B v_{n}-B z\right\| \\
\leq & \alpha_{n}\left\|f\left(x_{n}\right)-z\right\|^{2}+\left\|x_{n}-z\right\|^{2}-\delta_{n} \gamma_{n}\left\|u_{n}-M_{n}\right\|^{2} \\
& +2 \delta_{n} \gamma_{n} \alpha_{n}\left\|u_{n}-M_{n}\right\|\left\|A u_{n}-A z\right\|-\left(1-\delta_{n}\right) \gamma_{n}\left\|v_{n}-N_{n}\right\|^{2} \\
& +2\left(1-\delta_{n}\right) \gamma_{n} \eta_{n}\left\|v_{n}-N_{n}\right\|\left\|B v_{n}-B z\right\| .
\end{aligned}
$$

It follows that

$$
\begin{aligned}
\delta_{n} \gamma_{n}\left\|u_{n}-M_{n}\right\|^{2} \leq & \alpha_{n}\left\|f\left(x_{n}\right)-z\right\|^{2}+\left\|x_{n}-z\right\|^{2}-\left\|x_{n+1}-z\right\|^{2} \\
& +2 \delta_{n} \gamma_{n} \alpha_{n}\left\|u_{n}-M_{n}\right\|\left\|A u_{n}-A z\right\|-\left(1-\delta_{n}\right) \gamma_{n}\left\|v_{n}-N_{n}\right\|^{2} \\
& +2\left(1-\delta_{n}\right) \gamma_{n} \eta_{n}\left\|v_{n}-N_{n}\right\|\left\|B v_{n}-B z\right\| \\
\leq & \alpha_{n}\left\|f\left(x_{n}\right)-z\right\|^{2}+\left(\left\|x_{n}-z\right\|+\left\|x_{n+1}-z\right\|\right)\left\|x_{n+1}-x_{n}\right\| \\
& +2 \delta_{n} \gamma_{n} \alpha_{n}\left\|u_{n}-M_{n}\right\|\left\|A u_{n}-A z\right\|+2\left(1-\delta_{n}\right) \gamma_{n} \eta_{n}\left\|v_{n}-N_{n}\right\|\left\|B v_{n}-B z\right\| .
\end{aligned}
$$

From (3.17), (3.26), (3.27), and conditions (i)-(iii), we have

$$
\lim _{n \rightarrow \infty}\left\|u_{n}-M_{n}\right\|=0
$$

By using the same method as (3.50), we have

$$
\lim _{n \rightarrow \infty}\left\|v_{n}-N_{n}\right\|=0 .
$$


By (3.42) and (3.50), we have

$$
\lim _{n \rightarrow \infty}\left\|M_{n}-x_{n}\right\|=0
$$

By (3.43) and (3.51), we have

$$
\lim _{n \rightarrow \infty}\left\|N_{n}-x_{n}\right\|=0
$$

Since $M_{n}=P_{C}\left(u_{n}-\lambda_{n} A u_{n}\right)$ and $N_{n}=P_{C}\left(v_{n}-\eta_{n} B v_{n}\right)$, we have

$$
y_{n}-x_{n}=\delta_{n}\left(M_{n}-x_{n}\right)+\left(1-\delta_{n}\right)\left(N_{n}-x_{n}\right)
$$

By (3.52) and (3.53), we obtain

$$
\lim _{n \rightarrow \infty}\left\|y_{n}-x_{n}\right\|=0
$$

Note that

$$
\begin{aligned}
\left\|x_{n}-S_{n} x_{n}\right\| & \leq\left\|x_{n}-S_{n} y_{n}\right\|+\left\|S_{n} y_{n}-S_{n} x_{n}\right\| \\
& \leq\left\|x_{n}-S_{n} y_{n}\right\|+\left\|y_{n}-x_{n}\right\| .
\end{aligned}
$$

From (3.20) and (3.55), we have

$$
\lim _{n \rightarrow \infty}\left\|x_{n}-S_{n} x_{n}\right\|=0
$$

Step 5. We will show that

$$
\limsup _{n \rightarrow \infty}\left\langle f(z)-z, x_{n}-z\right\rangle \leq 0
$$

where $z=P_{\mathfrak{F}} f(z)$. To show this inequality, take subsequence $\left\{x_{n_{i}}\right\}$ of $\left\{x_{n}\right\}$ such that

$$
\limsup _{n \rightarrow \infty}\left\langle f(z)-z, x_{n}-z\right\rangle=\limsup _{i \rightarrow \infty}\left\langle f(z)-z, x_{n_{i}}-z\right\rangle .
$$

Since $\left\{x_{n_{i}}\right\}$ is bounded, there exists a subsequence $\left\{x_{n_{i j}}\right\}$ of $\left\{x_{n_{i}}\right\}$ which converges weakly to $q$. Without loss of generality, we can assume that $x_{n_{i}} \rightarrow q$. Since $C$ is closed convex, $C$ is weakly closed. So, we have $q \in C$. Let us show that $q \in \mathfrak{F}=\bigcap_{i=1}^{N} F\left(T_{i}\right) \cap \operatorname{EP}(F, A) \cap \operatorname{EP}(G, B) \cap$ $F\left(G_{1}\right) \cap F\left(G_{2}\right)$. We first show that $q \in \operatorname{EP}(F, A) \cap \operatorname{EP}(G, B) \cap F\left(G_{1}\right) \cap F\left(G_{2}\right)$. From (3.42), we have $u_{n_{i}} \rightarrow q$. Since $u_{n}=T_{r_{n}}\left(I-r_{n} A\right) x_{n}$, for any $y \in C$, we have

$$
F\left(u_{n}, y\right)+\left\langle A x_{n}, y-u_{n}\right\rangle+\frac{1}{r_{n}}\left\langle y-u_{n}, u_{n}-x_{n}\right\rangle \geq 0
$$


From (A2), we have

$$
\left\langle A x_{n}, y-u_{n}\right\rangle+\frac{1}{r_{n}}\left\langle y-u_{n}, u_{n}-x_{n}\right\rangle \geq F\left(y, u_{n}\right) .
$$

This implies that

$$
\left\langle A x_{n_{i}}, y-u_{n_{i}}\right\rangle+\frac{1}{r_{n_{i}}}\left\langle y-u_{n_{i}}, u_{n_{i}}-x_{n_{i}}\right\rangle \geq F\left(y, u_{n_{i}}\right)
$$

Put $z_{t}=t y+(1-t) q$ for all $t \in(0,1]$ and $y \in C$. Then, we have $z_{t} \in C$. So, from (3.62), we have

$$
\begin{aligned}
\left\langle z_{t}-u_{n_{i}}, A z_{t}\right\rangle \geq & \left\langle z_{t}-u_{n_{i}}, A z_{t}\right\rangle-\left\langle z_{t}-u_{n_{i}}, A x_{n_{i}}\right\rangle-\left\langle z_{t}-u_{n_{i}}, \frac{u_{n_{i}}-x_{n_{i}}}{r_{n_{i}}}\right\rangle+F\left(z_{t}, u_{n_{i}}\right) \\
= & \left\langle z_{t}-u_{n_{i}}, A z_{t}-A u_{n_{i}}\right\rangle+\left\langle z_{t}-u_{n_{i}}, A u_{n_{i}}-A x_{n_{i}}\right\rangle \\
& -\left\langle z_{t}-u_{n_{i}}, \frac{u_{n_{i}}-x_{n_{i}}}{r_{n_{i}}}\right\rangle+F\left(z_{t}, u_{n_{i}}\right) .
\end{aligned}
$$

Since $\left\|u_{n_{i}}-x_{n_{i}}\right\| \rightarrow 0$, we have $\left\|A u_{n_{i}}-A x_{n_{i}}\right\| \rightarrow 0$. Further, from monotonicity of $A$, we have $\left\langle z_{t}-u_{n_{i}}, A z_{t}-A u_{n_{i}}\right\rangle \geq 0$. So, from (A4), we have

$$
\left\langle z_{t}-q, A z_{t}\right\rangle \geq F\left(z_{t}, q\right) \quad \text { as } i \longrightarrow \infty .
$$

From (A1), (A4), and (3.64), we also have

$$
\begin{aligned}
0 & =F\left(z_{t}, z_{t}\right) \leq t F\left(z_{t}, y\right)+(1-t) F\left(z_{t}, q\right) \\
& \leq t F\left(z_{t}, y\right)+(1-t)\left\langle z_{t}-q, A z_{t}\right\rangle \\
& =t F\left(z_{t}, y\right)+(1-t) t\left\langle y-q, A z_{t}\right\rangle .
\end{aligned}
$$

Thus

$$
0 \leq F\left(z_{t}, y\right)+(1-t)\left\langle y-q, A z_{t}\right\rangle
$$

Letting $t \rightarrow 0$, we have, for each $y \in C$,

$$
0 \leq F(q, y)+\langle y-q, A q\rangle .
$$

This implies that

$$
q \in \operatorname{EP}(F, A)
$$


Fixed Point Theory and Applications

From (3.43), we have $v_{n i} \rightarrow q$. Since $v_{n}=T_{s_{n}}\left(I-s_{n} B\right) x_{n}$, for any $y \in C$, we have

$$
G\left(v_{n}, y\right)+\left\langle B x_{n}, y-v_{n}\right\rangle+\frac{1}{s_{n}}\left\langle y-v_{n}, v_{n}-x_{n}\right\rangle \geq 0
$$

From (A2), we have

$$
\left\langle B x_{n}, y-v_{n}\right\rangle+\frac{1}{s_{n}}\left\langle y-v_{n}, v_{n}-x_{n}\right\rangle \geq G\left(y, v_{n}\right) .
$$

This implies that

$$
\left\langle B x_{n_{i}}, y-v_{n_{i}}\right\rangle+\frac{1}{s_{n_{i}}}\left\langle y-v_{n_{i}}, v_{n_{i}}-x_{n_{i}}\right\rangle \geq G\left(y, v_{n_{i}}\right) .
$$

Put $z_{t}=t y+(1-t) q$ for all $t \in(0,1]$ and $y \in C$. Then, we have $z_{t} \in C$. So, from (3.71) we have

$$
\begin{aligned}
\left\langle z_{t}-v_{n_{i}}, B z_{t}\right\rangle \geq & \left\langle z_{t}-v_{n_{i}}, B z_{t}\right\rangle-\left\langle z_{t}-v_{n_{i}}, B x_{n_{i}}\right\rangle-\left\langle z_{t}-v_{n_{i}}, \frac{v_{n_{i}}-x_{n_{i}}}{s_{n_{i}}}\right\rangle+G\left(z_{t}, v_{n_{i}}\right) \\
= & \left\langle z_{t}-v_{n_{i}}, B z_{t}-B v_{n_{i}}\right\rangle+\left\langle z_{t}-v_{n_{i}}, B v_{n_{i}}-B x_{n_{i}}\right\rangle-\left\langle z_{t}-v_{n_{i}}, \frac{v_{n_{i}}-x_{n_{i}}}{s_{n_{i}}}\right\rangle \\
& +G\left(z_{t}, v_{n_{i}}\right) .
\end{aligned}
$$

Since $\left\|v_{n_{i}}-x_{n_{i}}\right\| \rightarrow 0$, we have $\left\|B v_{n_{i}}-B x_{n_{i}}\right\| \rightarrow 0$. Further, from monotonicity of $B$, we have $\left\langle z_{t}-v_{n_{i}}, B z_{t}-B v_{n_{i}}\right\rangle \geq 0$. So, from (A4), we have

$$
\left\langle z_{t}-q, B z_{t}\right\rangle \geq G\left(z_{t}, q\right)
$$

From (A1), (A4), and (3.64), we also have

$$
\begin{aligned}
0 & =G\left(z_{t}, z_{t}\right) \leq t G\left(z_{t}, y\right)+(1-t) G\left(z_{t}, q\right) \\
& \leq t G\left(z_{t}, y\right)+(1-t)\left\langle z_{t}-q, B z_{t}\right\rangle \\
& =t G\left(z_{t}, y\right)+(1-t) t\left\langle y-q, B z_{t}\right\rangle,
\end{aligned}
$$

hence

$$
0 \leq G\left(z_{t}, y\right)+(1-t)\left\langle y-q, B z_{t}\right\rangle .
$$

Letting $t \rightarrow 0$, we have, for each $y \in C$,

$$
0 \leq G(q, y)+\langle y-q, B q\rangle .
$$


This implies that

$q \in \operatorname{EP}(G, B)$.

Define a mapping $Q: C \rightarrow C$ by

$$
Q x=\delta P_{C}\left(I-\lambda_{n} A\right) x+(1-\delta) P_{C}\left(I-\eta_{n} B\right) x, \quad \forall x \in C,
$$

where $\lim _{n \rightarrow \infty} \delta_{n}=\delta \in(0,1)$. From Lemma 2.3, we have that $Q$ is nonexpansive with

$$
F(Q)=F\left(P_{C}\left(I-\lambda_{n} A\right)\right) \bigcap F\left(P_{C}\left(I-\eta_{n} B\right)\right) .
$$

Next, we show that

$$
\lim _{n \rightarrow n}\left\|x_{n}-Q x_{n}\right\|=0
$$

By nonexpansiveness of $I-\eta_{n} B$ and $I-\lambda_{n} A$, we have

$$
\begin{aligned}
& \left\|x_{n}-Q x_{n}\right\| \leq\left\|x_{n}-y_{n}\right\|+\left\|y_{n}-Q x_{n}\right\| \\
& =\left\|x_{n}-y_{n}\right\|+\| \delta_{n} P_{C}\left(u_{n}-\lambda_{n} A u_{n}\right)+\left(1-\delta_{n}\right) P_{C}\left(v_{n}-\eta_{n} B v_{n}\right)-\delta P_{C}\left(I-\lambda_{n} A\right) x_{n} \\
& -(1-\delta) P_{C}\left(I-\eta_{n} B\right) x_{n} \| \\
& =\left\|x_{n}-y_{n}\right\|+\| \delta_{n} P_{C}\left(I-\lambda_{n} A\right) u_{n}-\delta_{n} P_{C}\left(I-\lambda_{n} A\right) x_{n}+\delta_{n} P_{C}\left(I-\lambda_{n} A\right) x_{n} \\
& +\left(1-\delta_{n}\right) P_{C}\left(I-\eta_{n} B\right) v_{n}-\left(1-\delta_{n}\right) P_{C}\left(I-\eta_{n} B\right) x_{n} \\
& +\left(1-\delta_{n}\right) P_{C}\left(I-\eta_{n} B\right) x_{n}-\delta P_{C}\left(I-\lambda_{n} A\right) x_{n}-(1-\delta) P_{C}\left(I-\eta_{n} B\right) x_{n} \| \\
& =\left\|x_{n}-y_{n}\right\|+\| \delta_{n}\left(P_{C}\left(I-\lambda_{n} A\right) u_{n}-P_{C}\left(I-\lambda_{n} A\right) x_{n}\right)+\left(\delta_{n}-\delta\right) P_{C}\left(I-\lambda_{n} A\right) x_{n} \\
& +\left(1-\delta_{n}\right)\left(P_{C}\left(I-\eta_{n} B\right) v_{n}-P_{C}\left(I-\eta_{n} B\right) x_{n}\right) \\
& +\left(\delta-\delta_{n}\right) P_{C}\left(I-\eta_{n} B\right) x_{n} \| \\
& \leq\left\|x_{n}-y_{n}\right\|+\delta_{n}\left\|P_{C}\left(I-\lambda_{n} A\right) u_{n}-P_{C}\left(I-\lambda_{n} A\right) x_{n}\right\|+\left|\delta_{n}-\delta\right|\left\|P_{C}\left(I-\lambda_{n} A\right) x_{n}\right\| \\
& +\left(1-\delta_{n}\right)\left\|P_{C}\left(I-\eta_{n} B\right) v_{n}-P_{C}\left(I-\eta_{n} B\right) x_{n}\right\|+\left|\delta_{n}-\delta\right|\left\|P_{C}\left(I-\eta_{n} B\right) x_{n}\right\| \\
& \leq\left\|x_{n}-y_{n}\right\|+\delta_{n}\left\|u_{n}-x_{n}\right\|+\left|\delta_{n}-\delta\right|\left\|P_{C}\left(I-\lambda_{n} A\right) x_{n}\right\|+\left(1-\delta_{n}\right)\left\|v_{n}-x_{n}\right\| \\
& +\left|\delta_{n}-\delta\right|\left\|P_{C}\left(I-\eta_{n} B\right) x_{n}\right\| \\
& \leq\left\|x_{n}-y_{n}\right\|+\delta_{n}\left\|u_{n}-x_{n}\right\|+2\left|\delta_{n}-\delta\right| M_{1}+\left(1-\delta_{n}\right)\left\|v_{n}-x_{n}\right\|,
\end{aligned}
$$


where $M_{1}=\sup _{n \geq 0}\left\{\left\|P_{C}\left(I-\lambda_{n} A\right) x_{n}\right\|+\left\|P_{C}\left(I-\eta_{n} B\right) x_{n}\right\|\right\}$. From (3.17), (3.42), (3.43), (3.55), and condition (iii), we have $\lim _{n \rightarrow n}\left\|x_{n}-Q x_{n}\right\|=0$. Since $x_{n_{i}} \rightarrow q$, it follows from (3.80) that, $\lim _{i \rightarrow \infty}\left\|x_{n_{i}}-Q x_{n_{i}}\right\|=0$. By Lemma 2.4, we obtain that

$$
q \in F(Q)=F\left(P_{C}\left(I-\lambda_{n} A\right)\right) \cap F\left(P_{C}\left(I-\eta_{n} B\right)\right)=F\left(G_{1}\right) \cap F\left(G_{2}\right) .
$$

Assume that $q \neq S q$. Using Opial $s^{\prime}$ property, (3.57) and Lemma 2.10 we have

$$
\begin{aligned}
\liminf _{i \rightarrow \infty}\left\|x_{n_{i}}-q\right\| & <\liminf _{i \rightarrow \infty}\left\|x_{n_{i}}-S q\right\| \\
& \leq \liminf _{i \rightarrow \infty}\left(\left\|x_{n_{i}}-S_{n_{i}} x_{n_{i}}\right\|+\left\|S_{n_{i}} x_{n_{i}}-S_{n_{i}} q\right\|+\left\|S_{n_{i}} q-S q\right\|\right) \\
& \leq \liminf _{i \rightarrow \infty}\left\|x_{n_{i}}-q\right\| .
\end{aligned}
$$

This is a contradiction, so we have

$$
q \in \bigcap_{i=1}^{N} F\left(T_{i}\right)=F(S)
$$

From (3.68), (3.77) (3.82), and (3.84), we have $q \in \mathfrak{F}$. Since $P_{\mathfrak{F}} f$ is contraction with the coefficient $\theta \in(0,1), P_{\mathfrak{F}}$ has a unique fixed point. Let $z$ be a fixed point of $P_{\mathfrak{F}} f$, that is $z=P_{\mathfrak{F}} f(z)$. Since $x_{n_{i}} \rightarrow q$ and $q \in \mathfrak{F}$, we have

$$
\begin{aligned}
\limsup _{n \rightarrow \infty}\left\langle f(z)-z, x_{n}-z\right\rangle & =\limsup _{i \rightarrow \infty}\left\langle f(z)-z, x_{n_{i}}-z\right\rangle \\
& =\langle f(z)-z, q-z\rangle \leq 0 .
\end{aligned}
$$

Step 6. Finally, we will show that $x_{n} \rightarrow z$ as $n \rightarrow \infty$. By nonexpansiveness of $T_{r_{n}}, T_{S_{n}}, I-$ $\lambda_{n} A, I-\eta_{n} B, I-r_{n} A, I-s_{n} B$, we can show that $\left\|y_{n}-z\right\| \leq\left\|x_{n}-z\right\|$. Then

$$
\begin{aligned}
\left\|x_{n+1}-z\right\|^{2}= & \left\langle\alpha_{n}\left(f\left(x_{n}\right)-z\right)+\beta_{n}\left(x_{n}-z\right)+\gamma_{n}\left(S_{n} y_{n}-z\right), x_{n+1}-z\right\rangle \\
= & \alpha_{n}\left\langle f\left(x_{n}\right)-z, x_{n+1}-z\right\rangle+\beta_{n}\left\langle x_{n}-z, x_{n+1}-z\right\rangle+\gamma_{n}\left\langle S_{n} y_{n}-z, x_{n+1}-z\right\rangle \\
\leq & \alpha_{n}\left\langle f\left(x_{n}\right)-f(z), x_{n+1}-z\right\rangle+\alpha_{n}\left\langle f(z)-z, x_{n+1}-z\right\rangle+\beta_{n}\left\|x_{n}-z\right\|\left\|x_{n+1}-z\right\| \\
& +\gamma_{n}\left\|S_{n} y_{n}-z\right\| x_{n+1}-z \| \\
\leq & \alpha_{n}\left\|f\left(x_{n}\right)-f(z)\right\|\left\|x_{n+1}-z\right\|+\alpha_{n}\left\langle f(z)-z, x_{n+1}-z\right\rangle+\beta_{n}\left\|x_{n}-z\right\|\left\|x_{n+1}-z\right\| \\
& +\gamma_{n}\left\|y_{n}-z\right\|\left\|x_{n+1}-z\right\|
\end{aligned}
$$




$$
\begin{aligned}
\leq & \alpha_{n} \theta\left\|x_{n}-z\right\|\left\|x_{n+1}-z\right\|+\alpha_{n}\left\langle f(z)-z, x_{n+1}-z\right\rangle+\beta_{n}\left\|x_{n}-z\right\|\left\|x_{n+1}-z\right\| \\
& +\gamma_{n}\left\|x_{n}-z\right\|\left\|x_{n+1}-z\right\| \\
= & \left(1-\alpha_{n}(1-\theta)\right)\left\|x_{n}-z\right\|\left\|x_{n+1}-z\right\|+\alpha_{n}\left\langle f(z)-z, x_{n+1}-z\right\rangle \\
\leq & \left(1-\alpha_{n}(1-\theta)\right)\left(\frac{\left\|x_{n}-z\right\|^{2}+\left\|x_{n+1}-z\right\|^{2}}{2}\right)+\alpha_{n}\left\langle f(z)-z, x_{n+1}-z\right\rangle \\
\leq & \frac{\left(1-\alpha_{n}(1-\theta)\right)}{2}\left\|x_{n}-z\right\|^{2}+\frac{\left\|x_{n+1}-z\right\|^{2}}{2}+\alpha_{n}\left\langle f(z)-z, x_{n+1}-z\right\rangle ;
\end{aligned}
$$

we have

$$
\left\|x_{n+1}-z\right\|^{2} \leq\left(1-\alpha_{n}(1-\theta)\right)\left\|x_{n}-z\right\|^{2}+2 \alpha_{n}\left\langle f(z)-z, x_{n+1}-z\right\rangle
$$

By Step 5, (3.87), and Lemma 2.2, we have $\lim _{n \rightarrow \infty} x_{n}=z$, where $z=P_{\mathbb{F}} f(z)$. It easy to see that sequences $\left\{y_{n}\right\},\left\{u_{n}\right\}$, and $\left\{v_{n}\right\}$ converge strongly to $z=P_{\mathfrak{F}} f(z)$.

\section{Application}

Using our main theorem (Theorem 3.1), we obtain the following strong convergence theorems involving finite family of $\kappa$-strict pseudocontractions. as follows.

To prove strong convergence theorem in this section, we need definition and lemma

Definition 4.1. A mapping $T: C \rightarrow C$ is said to be a $\kappa$-strongly pseudo contraction mapping, if there exist $\kappa \in[0,1)$ such that

$$
\|T x-T y\|^{2} \leq\|x-y\|^{2}+\kappa\|(I-T) x-(I-T) y\|^{2}, \quad \forall x, y \in C .
$$

Lemma 4.2 (see [20]). Let $C$ be a nonempty closed convex subset of a real Hilbert space $H$ and $T: C \rightarrow C$ a $\kappa$-strict pseudo contraction. Define $S: C \rightarrow C$ by $S x=\alpha x+(1-\alpha)$ Tx for each $x \in C$. Then, as $\alpha \in[\kappa, 1) S$ is nonexpansive such that $F(S)=F(T)$.

Theorem 4.3. Let $C$ be a nonempty closed convex subset of a Hilbert space $H$. Let $F$ and $G$ be two bifunctions from $C \times C$ into $\mathbb{R}$ satisfying (A1)-(A4), respectively. Let $A: C \rightarrow H$ is a $\alpha$-inverse strongly monotone mapping and $B: C \rightarrow H$ be a $\beta$-inverse strongly monotone mapping. Let $\left\{T_{i}\right\}_{i=1}^{N}$ be a finite family of $\kappa_{i}$-psuedo contractions with $\mathfrak{F}=\bigcap_{i=1}^{N} F\left(T_{i}\right) \cap \operatorname{EP}(F, A) \cap E P(G, B) \cap F\left(G_{1}\right) \cap$ $F\left(G_{2}\right) \neq \emptyset$, where $G_{1}, G_{2}: C \rightarrow C$ are defined by $G_{1}(x)=P_{C}\left(x-\lambda_{n} A x\right), G_{2}(x)=P_{C}(x-$ $\left.\eta_{n} B x\right)$, for all $x \in C$. Define a mapping $T_{\mathcal{K}_{i}}$ by $T_{\kappa_{i}}=\kappa_{i} x+\left(1-\kappa_{i}\right) T_{i} x$, for all $x \in C, i \in\{1,2, \ldots, N\}$. Let $f: C \rightarrow C$ be a contraction with the coefficient $\theta \in(0,1)$. Let $S_{n}$ be the $S$-mappings generated by $T_{\kappa_{1}}, T_{\kappa_{2}}, \ldots, T_{\mathcal{K}_{N}}$ and $\alpha_{1}^{(n)}, \alpha_{2}^{(n)}, \ldots, \alpha_{N}^{(n)}$, where $\alpha_{j}^{(n)}=\left(\alpha_{1}^{n, j}, \alpha_{2}^{n, j}, \alpha_{3}^{n, j}\right) \in I \times I \times I, I=[0,1]$, 
$\alpha_{1}^{n, j}+\alpha_{2}^{n, j}+\alpha_{3}^{n, j}=1$ and $0<\eta_{1} \leq \alpha_{1}^{n, j} \leq \theta_{1}<1$ for all $n \in \mathbb{N}$, for all $j=1,2, \ldots, N-1,0<\eta_{N} \leq$ $\alpha_{1}^{n, N} \leq 1$ and $0 \leq \alpha_{2}^{n, j}, \alpha_{3}^{n, j} \leq \theta_{3}<1$ for all $n \in \mathbb{N}$, for all $j=1,2, \ldots, N$. Let $\left\{x_{n}\right\},\left\{u_{n}\right\},\left\{v_{n}\right\},\left\{y_{n}\right\}$ be sequences generated by $x_{1}, u, v \in C$

$$
\begin{array}{r}
F\left(u_{n}, u\right)+\left\langle A x_{n}, u-u_{n}\right\rangle+\frac{1}{r_{n}}\left\langle u-u_{n}, u_{n}-x_{n}\right\rangle \geq 0, \\
G\left(v_{n}, v\right)+\left\langle B x_{n}, v-v_{n}\right\rangle+\frac{1}{s_{n}}\left\langle v-v_{n}, v_{n}-x_{n}\right\rangle \geq 0, \\
y_{n}=\delta_{n} P_{C}\left(u_{n}-\lambda_{n} A u_{n}\right)+\left(1-\delta_{n}\right) P_{C}\left(v_{n}-\eta_{n} B v_{n}\right), \\
x_{n+1}=\alpha_{n} f\left(x_{n}\right)+\beta_{n} x_{n}+\gamma_{n} S_{n} y_{n}, \quad \forall n \geq 1,
\end{array}
$$

where $\left\{\alpha_{n}\right\},\left\{\beta_{n}\right\},\left\{\gamma_{n}\right\} \in(0,1)$ such that $\alpha_{n}+\beta_{n}+\gamma_{n}=1, r_{n} \in[a, b] \subset(0,2 \alpha), s_{n} \in[c, d] \subset$ $(0,2 \beta), \lambda_{n} \in[e, f] \subset(0,2 \alpha), \eta_{n} \in[g, h] \subset(0,2 \beta)$. Assume that

(i) $\lim _{n \rightarrow \infty} \alpha_{n}=0$ and $\sum_{n=0}^{\infty} \alpha_{n}=\infty$,

(ii) $\liminf _{n \rightarrow \infty} \beta_{n} \leq \lim \sup _{n \rightarrow \infty} \beta_{n}<1$,

(iii) $\lim _{n \rightarrow \infty} \delta_{n}=\delta \in(0,1)$,

(iv) $\sum_{n=0}^{\infty}\left|s_{n+1}-s_{n}\right|, \sum_{n=0}^{\infty}\left|r_{n+1}-r_{n}\right|, \sum_{n=0}^{\infty}\left|\lambda_{n+1}-\lambda_{n}\right|, \sum_{n=0}^{\infty}\left|\eta_{n+1}-\eta_{n}\right|, \sum_{n=0}^{\infty} \mid \alpha_{n+1}-$ $\alpha_{n}\left|, \quad \sum_{n=0}^{\infty}\right| \beta_{n+1}-\beta_{n} \mid<\infty$,

(v) $\left|\alpha_{1}^{n+1, j}-\alpha_{1}^{n, j}\right| \rightarrow 0$ and $\left|\alpha_{3}^{n+1, j}-\alpha_{3}^{n, j}\right| \rightarrow 0$ as $n \rightarrow \infty$, for all $j \in\{1,2,3, \ldots, N\}$.

Then the sequence $\left\{x_{n}\right\},\left\{y_{n}\right\},\left\{u_{n}\right\},\left\{v_{n}\right\}$ converges strongly to $z=P_{\mathfrak{F}} f(z)$, and $z$ is solution of

$$
\begin{aligned}
& \left\langle A x^{*}, x-x^{*}\right\rangle \geq 0, \\
& \left\langle B x^{*}, x-x^{*}\right\rangle \geq 0 .
\end{aligned}
$$

Proof. For every $i \in\{1,2, \ldots, N\}$, by Lemma 4.2, we have $T_{\kappa_{i}}$ is nonexpansive mappings. From Theorem 3.1, we can concluded the desired conclusion.

Theorem 4.4. Let $C$ be a nonempty closed convex subset of a Hilbert space $H$. Let $F$ and $G$ be two bifunctions from $C \times C$ into $\mathbb{R}$ satisfying (A1)-(A4), respectively. Let $A: C \rightarrow H$ be a $\alpha$-inverse strongly monotone mapping. Let $\left\{T_{i}\right\}_{i=1}^{N}$ be a finite family of $\kappa_{i}$-strict pseudo contractions with $\mathfrak{F}=$ $\bigcap_{i=1}^{N} F\left(T_{i}\right) \cap \operatorname{EP}(F, A) \cap F\left(G_{1}\right) \neq \emptyset$, where $G_{1}: C \rightarrow C$ defined by $G_{1}(x)=P_{C}\left(x-\lambda_{n} A x\right)$, for all $x \in$ C. Define a mapping $T_{\kappa_{i}}$ by $T_{\kappa_{i}}=\kappa_{i} x+\left(1-\kappa_{i}\right) T_{i} x$, for all $x \in C, i \in \mathbb{N}$. Let $f: C \rightarrow C$ a contraction with the coefficient $\theta \in(0,1)$. Let $S_{n}$ be the S-mappings generated by $T_{\kappa_{1}}, T_{\kappa_{2}}, \ldots, T_{\mathcal{K}_{N}}$ and $\alpha_{1}^{(n)}, \alpha_{2}^{(n)}, \ldots, \alpha_{N}^{(n)}$, where $\alpha_{j}^{(n)}=\left(\alpha_{1}^{n, j}, \alpha_{2}^{n, j}, \alpha_{3}^{n, j}\right) \in I \times I \times I, I=[0,1], \alpha_{1}^{n, j}+\alpha_{2}^{n, j}+\alpha_{3}^{n, j}=1$ and $0<\eta_{1} \leq \alpha_{1}^{n, j} \leq \theta_{1}<1$ for all $n \in \mathbb{N}$, for all $j=1,2, \ldots, N-1,0<\eta_{N} \leq \alpha_{1}^{n, N} \leq 1$ and 
$0 \leq \alpha_{2}^{n, j}, \alpha_{3}^{n, j} \leq \theta_{3}<1$ for all $n \in \mathbb{N}$, for all $j=1,2, \ldots, N$. Let $\left\{x_{n}\right\},\left\{u_{n}\right\},\left\{y_{n}\right\}$ be sequences generated by $x_{1}, u, \in C$

$$
\begin{gathered}
F\left(u_{n}, u\right)+\left\langle A x_{n}, u-u_{n}\right\rangle+\frac{1}{r_{n}}\left\langle u-u_{n}, u_{n}-x_{n}\right\rangle \geq 0, \\
y_{n}=P_{C}\left(u_{n}-\lambda_{n} A u_{n}\right), \\
x_{n+1}=\alpha_{n} f\left(x_{n}\right)+\beta_{n} x_{n}+\gamma_{n} S_{n} y_{n}, \quad \forall n \geq 1,
\end{gathered}
$$

where $\left\{\alpha_{n}\right\},\left\{\beta_{n}\right\},\left\{\gamma_{n}\right\} \in(0,1)$ such that $\alpha_{n}+\beta_{n}+\gamma_{n}=1, r_{n} \in[a, b] \subset(0,2 \alpha), \lambda_{n} \in[e, f] \subset$ $(0,2 \alpha)$. Assume that

(i) $\lim _{n \rightarrow \infty} \alpha_{n}=0$ and $\sum_{n=0}^{\infty} \alpha_{n}=\infty$,

(ii) $0<\liminf _{n \rightarrow \infty} \beta_{n} \leq \limsup _{n \rightarrow \infty} \beta_{n}<1$,

(iii) $\sum_{n=0}^{\infty}\left|r_{n+1}-r_{n}\right|, \sum_{n=0}^{\infty}\left|\lambda_{n+1}-\lambda_{n}\right|, \sum_{n=0}^{\infty}\left|\alpha_{n+1}-\alpha_{n}\right|, \sum_{n=0}^{\infty}\left|\beta_{n+1}-\beta_{n}\right|<\infty$,

(iv) $\left|\alpha_{1}^{n+1, j}-\alpha_{1}^{n, j}\right| \rightarrow 0$ and $\left|\alpha_{3}^{n+1, j}-\alpha_{3}^{n, j}\right| \rightarrow 0$ as $n \rightarrow \infty$, for all $j \in\{1,2,3, \ldots, N\}$.

Then the sequence $\left\{x_{n}\right\},\left\{y_{n}\right\},\left\{u_{n}\right\}$ converges strongly to $z=P_{\mathfrak{F}} f(z)$, and $z$ is solution of

$$
\left\langle A x^{*}, x-x^{*}\right\rangle \geq 0
$$

Proof. For every $i \in\{1,2, \ldots, N\}$, by Lemma 4.2, we have that $T_{\kappa_{i}}$ is nonexpansive mappings, putting $F \equiv G, A \equiv B, s_{n}=r_{n}, \lambda_{n}=\eta_{n}$, and $u_{n}=v_{n}$. From Theorem 3.1, we can conclude the desired conclusion.

\section{Acknowledgment}

The authors would like to thank Professor Dr. Suthep Suantai for his suggestion in doing and improving this paper.

\section{References}

[1] K. Goebel and W. A. Kirk, Topics in Metric Fixed Point Theory, vol. 28 of Cambridge Studies in Advanced Mathematics, Cambridge University Press, Cambridge, UK, 1990.

[2] E. Blum and W. Oettli, "From optimization and variational inequalities to equilibrium problems," The Mathematics Student, vol. 63, no. 1-4, pp. 123-145, 1994.

[3] P. L. Combettes and S. A. Hirstoaga, "Equilibrium programming in Hilbert spaces," Journal of Nonlinear and Convex Analysis, vol. 6, no. 1, pp. 117-136, 2005.

[4] A. Moudafi and M. Théra, "Proximal and dynamical approaches to equilibrium problems," in IllPosed Variational Problems and Regularization Techniques, vol. 477 of Lecture Notes in Econom. and Math. Systems, pp. 187-201, Springer, Berlin, Germany, 1999.

[5] H. Iiduka and W. Takahashi, "Weak convergence theorems by Cesáro means for nonexpansive mappings and inverse-strongly-monotone mappings," Journal of Nonlinear and Convex Analysis, vol. 7, no. 1, pp. 105-113, 2006.

[6] H. H. Bauschke and J. M. Borwein, “On projection algorithms for solving convex feasibility problems," SIAM Review, vol. 38, no. 3, pp. 367-426, 1996.

[7] P. L. Combettes, "The foundations of set theoretic estimation," Proceedings of the IEEE, vol. 81, pp. 182-208, 1993. 
[8] A. Kangtunyakarn and S. Suantai, "A new mapping for finding common solutions of equilibrium problems and fixed point problems of finite family of nonexpansive mappings," Nonlinear Analysis: Theory, Methods \& Applications, vol. 71, no. 10, pp. 4448-4460, 2009.

[9] V. Colao, G. Marino, and H.-K. Xu, "An iterative method for finding common solutions of equilibrium and fixed point problems," Journal of Mathematical Analysis and Applications, vol. 344, no. 1, pp. 340352, 2008.

[10] S. Takahashi and W. Takahashi, "Viscosity approximation methods for equilibrium problems and fixed point problems in Hilbert spaces," Journal of Mathematical Analysis and Applications, vol. 331, no. 1, pp. 506-515, 2007.

[11] S. Takahashi and W. Takahashi, "Strong convergence theorem for a generalized equilibrium problem and a nonexpansive mapping in a Hilbert space," Nonlinear Analysis: Theory, Methods E Applications, vol. 69, no. 3, pp. 1025-1033, 2008.

[12] X. Qin, Y. J. Cho, and S. M. Kang, "Viscosity approximation methods for generalized equilibrium problems and fixed point problems with applications," Nonlinear Analysis: Theory, Methods $\mathcal{E}$ Applications, vol. 72, no. 1, pp. 99-112, 2010.

[13] W. Takahashi, Nonlinear Functional Analysis: Fixed Point Theory and Its Applications, Yokohama Publishers, Yokohama, Japan, 2000.

[14] H.-K. Xu, "Iterative algorithms for nonlinear operators," Journal of the London Mathematical Society. Second Series, vol. 66, no. 1, pp. 240-256, 2002.

[15] R. E. Bruck Jr., "Properties of fixed-point sets of nonexpansive mappings in Banach spaces," Transactions of the American Mathematical Society, vol. 179, pp. 251-262, 1973.

[16] F. E. Browder, "Nonlinear operators and nonlinear equations of evolution in Banach spaces," in Proceedings of Symposia in Pure Mathematics, pp. 1-308, Amer. Math. Soc., Providence, RI, USA, 1976.

[17] T. Suzuki, "Strong convergence of Krasnoselskii and Mann's type sequences for one-parameter nonexpansive semigroups without Bochner integrals," Journal of Mathematical Analysis and Applications, vol. 305, no. 1, pp. 227-239, 2005.

[18] A. Kangtunyakarn and S. Suantai, "Hybrid iterative scheme for generalized equilibrium problems and fixed point problems of finite family of nonexpansive mappings," Nonlinear Analysis: Hybrid Systems, vol. 3, no. 3, pp. 296-309, 2009.

[19] W. Takahashi, Introduction to Nonlinear and Convex Analysis, Yokohama Publishers, Yokohama, Japan, 2009.

[20] H. Zhou, "Convergence theorems of fixed points for $\kappa$-strict pseudo-contractions in Hilbert spaces," Nonlinear Analysis: Theory, Methods \& Applications, vol. 69, no. 2, pp. 456-462, 2008. 